\title{
An integration of enhanced social force and crowd control models for high-density crowd simulation
}

\author{
Hoshang Kolivand ${ }^{1,2}$ (D) Mohd Shafry Rahim ${ }^{1} \cdot$ Mohd Shahrizal Sunar ${ }^{1} \cdot$ Ahmad Zakwan Azizul Fata $^{1} \cdot$ \\ Chris Wren ${ }^{2}$
}

Received: 23 October 2019/Accepted: 24 September 2020/Published online: 9 October 2020

(C) The Author(s) 2020

\begin{abstract}
Social force model is one of the well-known approaches that can successfully simulate pedestrians' movements realistically. However, it is not suitable to simulate high-density crowd movement realistically due to the model having only three basic crowd characteristics which are goal, attraction, and repulsion. Therefore, it does not satisfy the high-density crowd condition which is complex yet unique, due to its capacity, density, and various demographic backgrounds of the agents. Thus, this research proposes a model that improves the social force model by introducing four new characteristics which are gender, walking speed, intention outlook, and grouping to make simulations more realistic. Besides, the high-density crowd introduces irregular behaviours in the crowd flow, which is stopping motion within the crowd. To handle these scenarios, another model has been proposed that controls each agent with two different states: walking and stopping. Furthermore, the stopping behaviour was categorized into a slow stop and sudden stop. Both of these proposed models were integrated to form a high-density crowd simulation framework. The framework has been validated by using the comparison method and fundamental diagram method. Based on the simulation of 45,000 agents, it shows that the proposed framework has a more accurate average walking speed $(0.36 \mathrm{~m} / \mathrm{s})$ compared to the conventional social force model $(0.61 \mathrm{~m} / \mathrm{s})$. Both of these results are compared to the real-world data which is $0.3267 \mathrm{~m} / \mathrm{s}$. The findings of this research will contribute to the simulation activities of pedestrians in a highly dense population.
\end{abstract}

Keywords High-density crowd simulation $\cdot$ Crowd simulation $\cdot$ Crowd control models $\cdot$ Social force model

\section{Introduction}

Animating motion for high-density crowds is a hard task to be completed. Most researchers used one of the main three popular approaches to simulate the high-density crowds including cellular automata model, rule-based model, and social forces model. Many works from many researchers have been done to improve the realism of the simulation [43]. However, these current models are not capable of realistic simulation of high-density crowds [2, 15]. The

Hoshang Kolivand

H.Kolivand@ljmu.ac.uk

1 MaGIC-X (Media and Games Innovation Centre of Excellence), UTM-IRDA Digital Media Centre Universiti Teknologi Malaysia, 81310 Skudai Johor, Malaysia

2 Department of Computer Science, Liverpool John Moores University, L3 3AF Liverpool, UK fastest and the least expensive computation is the cellular automata technique by specific strength of using a parallel computational model which integrates macroscopic and microscopic behaviours [42].

Yet, each agent can only move in eight directions. As a result, the simulation will look like a checkerboard especially in high-density crowd [30, 40]. The rule-based model is also comparatively fast showing different behaviours in simulating high-density crowds [49]. However, this model does not contain collision detection [1]. As a result, there are no repulsion forces between agents, which might be acceptable if the size of the crowd is small. However, if the size of the crowd is huge, agents might collide with each other and make it unrealistic [12]. For that reason, rulebased model is not suitable to simulate high-density crowd population. The social force model can simulate a crowd successfully for small or medium-size groups such as metro station [8, 24]. However, it also degrades the performance 
when the size of the crowd increases [51]. The increase in populations in the crowd makes the resulting simulation unrealistic since all the agents within the crowd will be moving at the same flow $[27,45]$. These current models for high-density crowd simulation cannot realistically handle body-to-body contact for large crowds, and while they achieve good results for low and medium density crowds in normal situations (e.g. people walking in a small town or in a park), they fail to realistically simulate high-density situations.

High-density crowd agents come from various backgrounds, which indirectly affect their behaviour, such as walking speed, genders, and intention outlook. However, these three characteristics are mostly ignored by other researchers. Most researchers treat each agent as the same individual, which means they will have the same walking speed, genders, and intention outlook [17]. This will make the crowd movement unrealistic since in a high-density crowd agents tend to change their walking direction and speed more often than agents in a sparse crowd [22] mainly when using data-driven methods [46]. Thus, a proper highdensity crowd simulation needs to handle these kinds of behaviours.

Most high-density crowd models simulate crowds as individual agents and not as a group. The existence of family and friendship groups within the crowd is overlooked by many researchers [11]. Eliyan et al. [11] presented a state-based model for group behaviour by associating some well-known activities. The grouping behaviour may lead to a dynamic crowd flow $[20,31,33,38]$, and it is the core issue of crowd simulation [48]. For example, the agents in groups tend to move together towards the same direction which causes the other agents not in the group to make way for the agent within the group [48]. For that reason, the techniques which consider the agents only as individuals cannot be generalized for the agents that move in groups.

Although bottleneck effect and arc formation are able to simulate the stopping motion realistically, in high-density scenarios such as Tawaf, the lack of walls and doors surrounding the crowds cause both models not suitable to be implemented. However, stopping behaviour still occurs within high-density crowd due to congestion, slow walking speed, and indecisive decision of the agents [22]. Chen et al. [8] tried to establish a bidirectional pedestrian flow model by considering only two main factors. They are the impacts between pedestrians and conflict frequency. This behaviour will affect the trajectories of other agents whether to stop, slow down, or take another path [26].

Recently, Salehi and Sung [35] presented a velocitybased technique for simulating crowd when some of the agents move in a specific formation. The main strength of this work is considering group movement and collision (which are a part of our technique) using optimal reciprocal collision avoidance. The main issues with the presented method can be feasibility if the obstacles are static. Moreover, they focused on the scenarios that groups try to maintain the group formation.

The primary goal of this research is to design a highdensity crowd simulation framework that increases the realism of high-density crowd simulation, while people move in groups or individuals such as Tawaf.

\section{Method}

The social force model is enhanced in developing crowd simulation. The social force model has been chosen because it is one of the most adaptable crowd simulation models to be developed [20]. This model will be modified and enhanced in order to derive a novel model to improve the realism of the high-density crowd simulation.

In crowd control model, the pedestrian "stopping" behaviour is developed. Generally, in a large and dense crowd situation, agents stopping, or stalling occurs commonly. Agents have the tendency to stop in the middle of their path due to several factors; other agents blocking their path, their paths collide with other people's paths or the area is too crowded to move. Thus, it is important that this behaviour is included in this research.

After both crowd simulation model and crowd control model are completed, these two models need to be integrated so that it can produce a novel high-density crowd simulation framework.

\subsection{Social force model}

Social force model just assumes that everyone within the crowd has the same characteristics, which make the pedestrians within the crowd simulation move in a uniform pattern without any hectic behaviour of a typical crowd. To manage this problem, we have introduced a new concept called a Zahmah.

Pedestrians in crowd simulation are called agents. Each agent position is defined in two dimensions: $\mathrm{x}$-axis and $\mathrm{y}$ axis. As an example, for agent $\alpha$, the agent's position $\boldsymbol{p}_{\alpha}$ is represented on the $\mathrm{x}$-axis as $x_{\alpha}$ and the position on the $\mathrm{y}$ axis as $y_{\alpha}$. Agents do not have the third dimension because they mostly just walk on a flat surface. (A full description of symbols is presented in "Appendix".)

The main force in social force model is the driving force, $\vec{f}_{\text {drive }}$. This force is described as the motivation of the agent $\alpha$ to move towards the desired direction $d_{\alpha}$. The agent will move according to his or her desired walking velocity, $\overrightarrow{\boldsymbol{v}}_{\alpha}$, while $v_{a}^{d}$ is the deviation of $\overrightarrow{\boldsymbol{v}}_{\alpha}$, where $\overrightarrow{\boldsymbol{v}}_{c}$ is the actual 
walking velocity of the agents and $\tau_{a}$ is the relaxation time for agent $\alpha$. Relaxation time is the finite amount of time that is required for agents to react and physically change their velocity $[14,16,35,50]$.

To get the desired direction, $\vec{d}_{\alpha}$ of agent $\alpha$ is by calculating the distance between destination or goal position of agent $\alpha, \overrightarrow{\boldsymbol{g}}_{\alpha}$ and the current position of agent $\alpha, \overrightarrow{\boldsymbol{p}}_{\alpha}$. Goal position is the main objective which motivates agent $\alpha$ to walk, while the current position is the position of agent $\alpha$ at that time. Since $\overrightarrow{\boldsymbol{g}}_{\alpha}$ and $\overrightarrow{\boldsymbol{p}}_{\alpha}$ are both vector values, the use of double modulus is more suitable to represent the norm value $[14,50]$.

The second force is the repulsion forces between agents within the crowd. This force is important so that the agents do not collide with each other. Each agent will have their own, repulsion strength. The strength depends on the distance between agents with another agent in that scenario, for example the position of agent $\alpha \overrightarrow{\boldsymbol{p}}_{\alpha}$ and position of agent $\beta \overrightarrow{\boldsymbol{p}}_{\beta}$. Each agent also will have their radius of interaction, $r_{\alpha}$ and $r_{\beta}$, respectively. This determines the distance for each interaction start. Lastly, $\overrightarrow{\boldsymbol{n}}_{a \beta}$ is the normalized vector pointing from agent $\alpha$ towards agent $\beta$.

In conclusion, the social force model consists of three forces that work together to determine the next position of the agent during that period. These forces are driving force $\vec{f}_{\text {drive }}$, repulsion force, $\vec{f}_{\text {repulsion, and attraction force, }}$ $\vec{f}_{\text {attraction. }}$ This is a continuous process until all the agents arrived at their goal position. This sum of all forces $\vec{f}_{\text {social }}$ describes the movement and direction of the agent as shown in Eq. $1[14,50]$. Goal position is the main objective which motivates agent $\alpha$ to walk, while the current position is the position of agent $\alpha$ at that time. In order to get the desired direction towards the goal (Fig. 1), the distance between destination or goal position of agent $\alpha g_{a}$ and the current position of agent $\alpha p_{a}$ needs to be calculated $\left(\vec{g}_{\alpha}-\vec{p}_{\alpha} /\left|\vec{g}_{\alpha}-\vec{p}_{\alpha}\right|\right)$ which | | represents the magnitude of related vectors. Moreover, deviation of walking speed of the agent is the factor to transmit the agent to the desired



Fig. 1 Driving force for SFM destination. This is while the actual walking velocity has not started from 0 . Figure 1 shows this concept.

In terms of repulsion force between two objects $\alpha$ and $\beta$, it is exponential with the direct proportion of the distance between two agents and inversely proportional with the radius of interaction for the agents. This amount must be calculated in the direction of agent $\alpha$ towards agent $\beta\left(\vec{n}_{a \beta}\right)$.

The last force contributing in SFM is attraction force which is in the same direction of repulsion force but with the magnitude of a constant called $C$ which is a scaling parameter of attraction forces between agents from the same group

$$
\begin{aligned}
\vec{f}_{\text {social }}= & \vec{f}_{\text {drive }}+\sum_{\alpha=1}^{n} \vec{f}_{\text {repulsion }}+\sum_{\alpha=1}^{n} \vec{f}_{\text {attraction }} \\
= & \frac{v_{a}^{d}\left(\frac{\vec{g}_{\alpha}-\vec{p}_{\alpha}}{\left|\vec{g}_{\alpha}-\vec{p}_{\alpha}\right|}\right)-\vec{v}_{c}}{\tau_{a}}+\sum_{\alpha=1}^{n} \exp \left(\frac{\left|\vec{p}_{\alpha}-\vec{p}_{\beta}\right|}{r}\right) \vec{n}_{a \beta} \\
& +\sum_{\alpha=1}^{n} c\left(\vec{n}_{a \beta}\right)
\end{aligned}
$$

As can be seen in Fig. 2 in standard SFM, agent $\alpha$ moves towards his or her destination by driving force and interacts with agent $\beta$ via interaction force. This is while in the presented model.

The second force is the repulsion forces between agents within the crowd. This force is important so that the agents do not collide with each other. Each agent will have their own, repulsion strength. The strength depends on the distance between agents with another agent in that scenario, for example the position of agent $\alpha \vec{p}_{\alpha}$ and position of agent $\beta \vec{p}_{\beta}$, which is shown in Fig. 3.

Our new presented model extends the existing social force model by making the three main forces as the fundamental forces, which are the driving force, repulsion force and attraction force (Fig. 4 (left)).

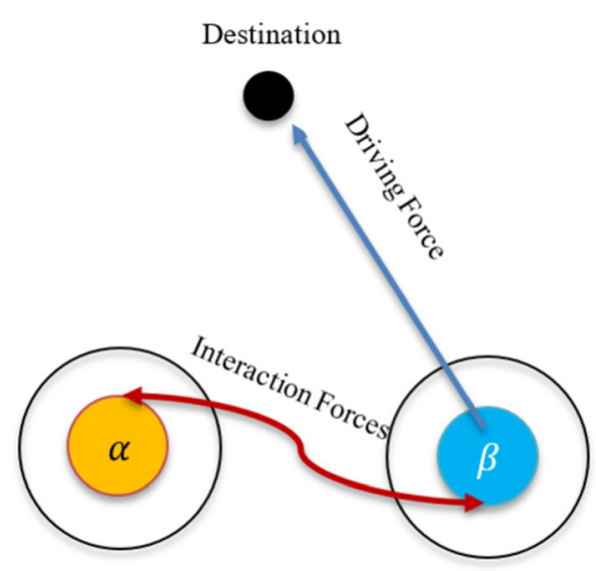

Fig. 2 Basis forces of social force model 
Fig. 3 Repulsion forces between agents
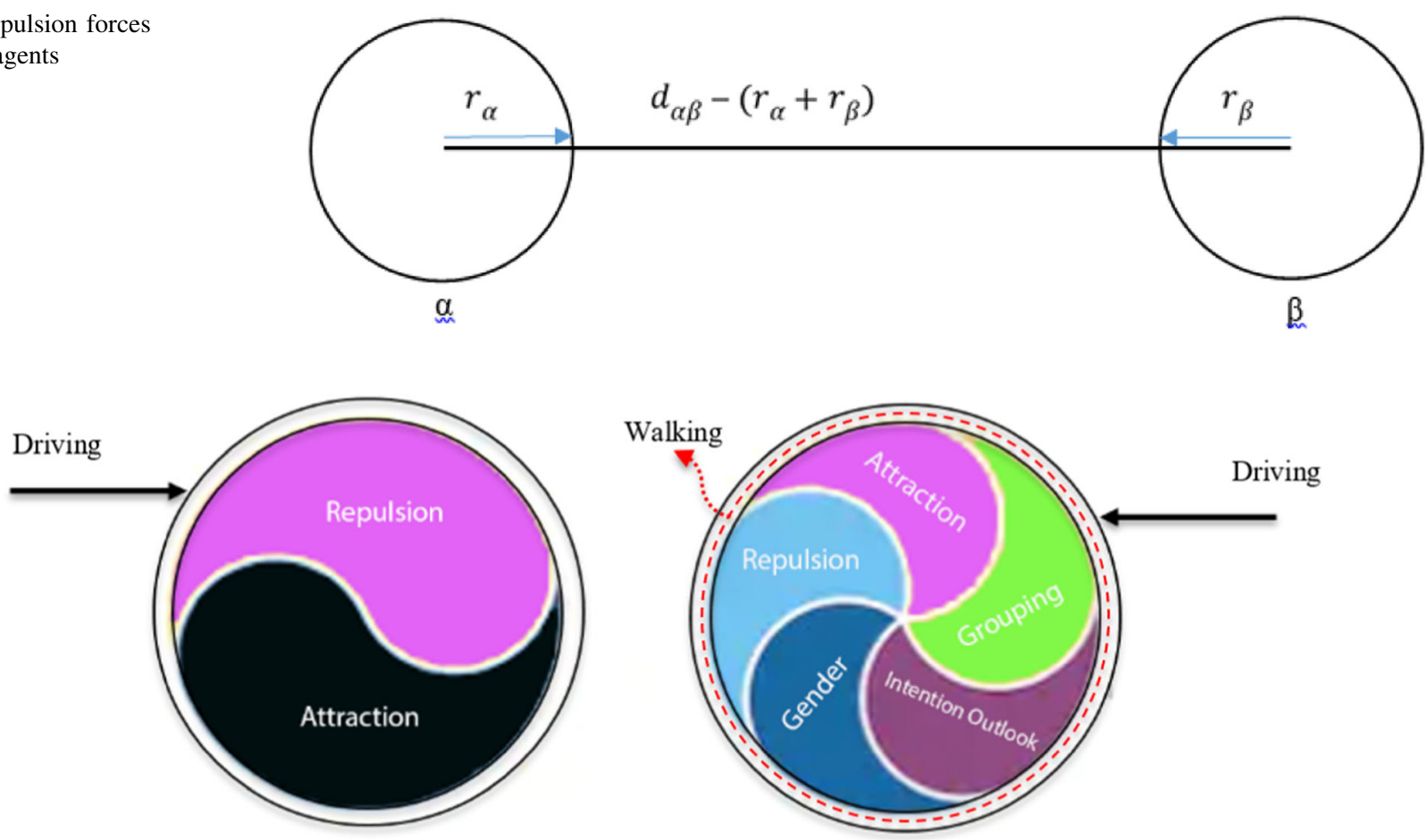

Fig. 4 (left) Basic social force model forces, (right) proposed model

The new model is presented to improve the social force model which is called Zahmah Social Force Model (ZFSM) (Fig. 4(right)). The ZSFM extends the existing social force model by making the three main forces as the fundamental forces, which are the driving force, repulsion force, and attraction force. The time complexity for the standard social force model as can be seen from Eq. 1 is $\sum_{1}^{n}(O(1)+O(n)+O(n))=O\left(n^{2}\right)$, which later we will see it is the same as our presented method.

One of the new forces of ZSFM is the intention outlook force. The intention of outlook or goal is the desired position that each agent wants to go. The goal position is different for each agent.

In a simple crowd simulation, the goal positions are normally linear; thus, agents will walk directly towards their intended position. However, during Tawaf, agents need to circle around the Kaabah, thus making it the epicentre of the circumambulation, t. Other than that since the agents are moving around the Kaabah, his/her position is the goal position of the agents. Thus, in ZSFM the goal position, $\overrightarrow{\boldsymbol{g}}_{a}$ will constantly move around the Kaabah to make the agents circle around the Kaabah (Eq. 2 and Fig. 5). Parameter $\mathrm{d}$ is the distance between the agent's goal position $\overrightarrow{\boldsymbol{g}}_{a}$ from their current position $\overrightarrow{\boldsymbol{p}}_{a}$. The smaller the distance value (d), the more accurate the rotation calculation around the Kaabah will be, since the movement will be more circular.

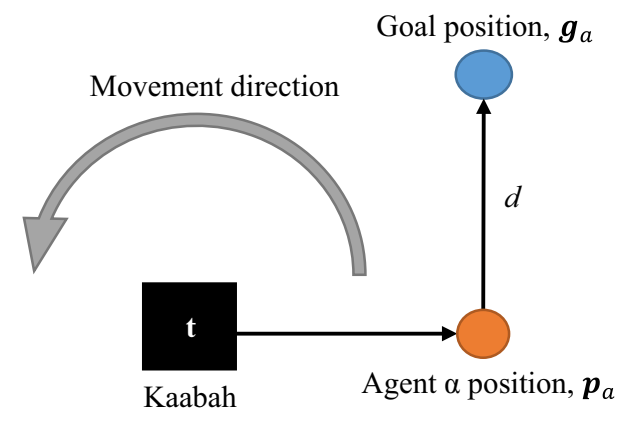

Fig. 5 Movement of agent's position and goal position

$\overrightarrow{\boldsymbol{g}}_{a}=\overrightarrow{\boldsymbol{p}}_{a}+\frac{d\left(\overrightarrow{\boldsymbol{t}}-\overrightarrow{\boldsymbol{p}}_{a}\right) \times \overrightarrow{\boldsymbol{Y}}}{\left|\left(\overrightarrow{\boldsymbol{t}}-\overrightarrow{\boldsymbol{p}}_{a}\right) \times \overrightarrow{\boldsymbol{Y}}\right|}$

Gender plays an important role in crowd simulation. This is because it will influence the behaviour of the agent in terms of the interaction with other agents especially in our case study (Tawaf). In a basic crowd simulation, the goal positions are normally linear, and agents will walk directly towards their intended position. However, during Tawaf, there are several rules that need to be followed by each pedestrian. One of these rules is that agents with a different gender cannot touch each other. If they touched each other, both of them have to restart their Tawaf ritual all over again. Thus, pedestrians will try to avoid each other if they encounter different gender agents. 
None of the previous researchers is focusing on simulating the different interactions between both genders. This is because mostly in other situations there are only minor differences between both interactions [17, 25]. However, in Tawaf scenario, it plays a very important part to simulate the situation precisely [36]. In order to simulate this situation, the interaction force equation has been modified to make it more suitable to be used in ZSFM. The repulsion force from the social force model is modified by introducing a new scalar parameter, $s$ (Eq. 3), which controls the strength of the repulsion forces. For example, if two different gender agents meet, the value of the scalar parameter $s$ will be increased compared to where two agents with the same gender meet.

$\vec{f}_{\text {gender }}=s\left(\exp \left[\frac{\left|\overrightarrow{\boldsymbol{p}}_{\alpha}-\overrightarrow{\boldsymbol{p}}_{\beta}\right|}{r}\right] \overrightarrow{\boldsymbol{n}}_{a \beta}\right)$

To understand the presented concept of Eq. 3, Tables 1 and 2 are provided with three different experiments to show the repulsion threshold and strength, respectively. As can be seen in Table 1, each agent has its own repulsion forces and repulsion threshold. Repulsion strength is the interaction strength towards other agents so that they do not collide with each other. Meanwhile, repulsion threshold is the distance in which the interaction forces will begin to trigger. If different genders pedestrians meet with each other, both of them will have a higher repulsion force compared to if they have the same gender. However, their repulsion threshold will remain the same for both interactions. The repulsion threshold remains the same because

Table 1 Repulsion threshold experiment

\begin{tabular}{lll}
\hline First Experiment & Second experiment & Third experiment \\
\hline$D 1=1.00 \mathrm{f}$ & $D 1=2.0 \mathrm{f}$ & $D 1=3.0 \mathrm{f}$ \\
$D 2=0.50 \mathrm{f}$ & $D 2=1.0 \mathrm{f}$ & $D 2=2.0 \mathrm{f}$ \\
$D 3=0.25 \mathrm{f}$ & $D 3=0.5 \mathrm{f}$ & $D 3=1.0 \mathrm{f}$
\end{tabular}
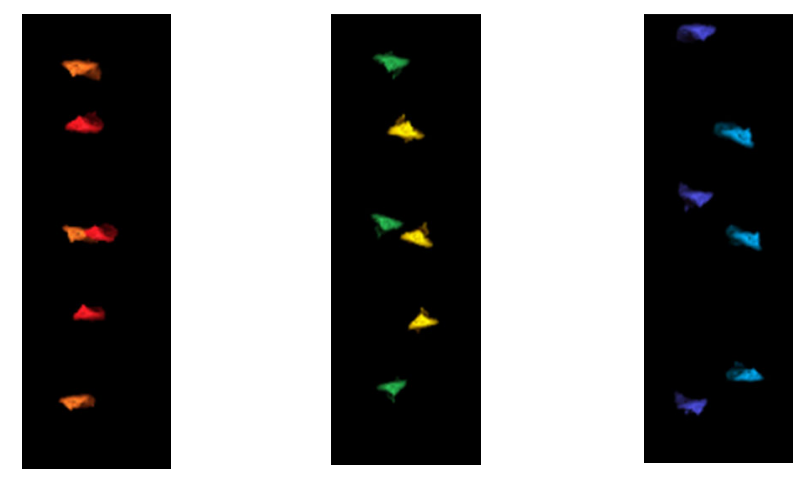

Table 2 Repulsion strength experiment

\begin{tabular}{lll}
\hline First experiment & Second experiment & Third experiment \\
\hline$F 1=0.0011$ & $F 1=0.015$ & $F 1=0.019$ \\
$F 2=0.0001$ & $F 2=0.005$ & $F 2=0.009$ \\
$F 3=0.0021$ & $F 3=0.0025$ & $F 3=0.0029$
\end{tabular}
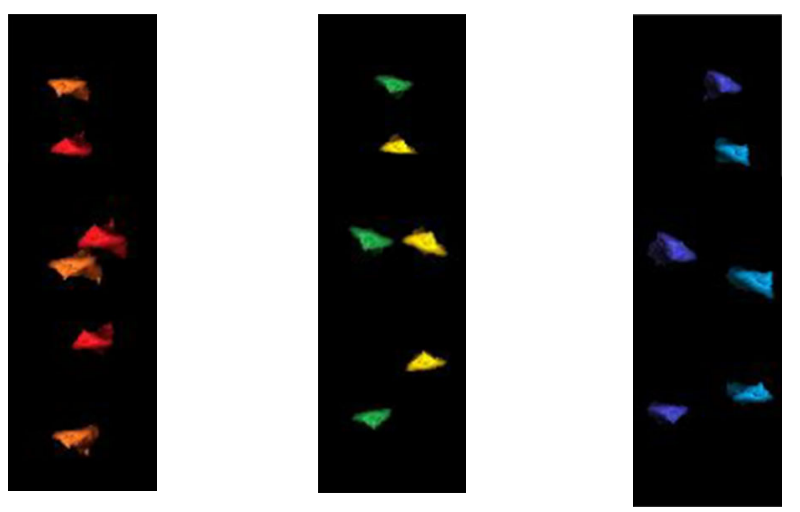

people mostly have a similar distance of interaction even though the repulsion force is different [6, 37]. The interaction forces also increase for closer agents towards each other [18] because to avoid them from colliding with each other. Moreover, on the contrary of basic SFM, in our case, agents need to circle around the Kaabah, making it the epicentre of the circumambulation, $\vec{t}$.

To get the optimal repulsion strength for both genders, a similar approach is being used. However, the repulsion strength is being manipulated instead of the repulsion threshold. The repulsion threshold values remain the same for every experiment $(D 1=2.0 \mathrm{f}, D 2=1.0 \mathrm{f}, D 3=0.5 \mathrm{f})$. $F 1$ represents the repulsion strength when the agent is closest towards each other, while $F 3$ the farthest and $F 2$ in between. Like the repulsion experiment, this experiment is done by making two agents walk towards each other and see the optimal repulsion strength to be used in high-density crowd simulation. Based on the experiment results in Table 2, the optimal repulsion strength values are the second experiment $\quad(F 1=0.019, \quad F 2=0.009$, $F 3=0.0029)$. This is because in the first experiment, the repulsion strength is too weak which makes the agents collide with each other, while the third experiment the repulsion strength is too strong, which makes the agents to avoid closing by too much. However, the third experiment values $(F 1=0.019, F 2=0.009, F 3=0.0029)$ are suitable to be used as the opposite gender repulsion strength, since stronger strength values make the agent interaction awkward and unnatural. Thus, this research uses the second 
and third experiment repulsion strength values as the same gender and different gender repulsion strength, respectively.

Everybody has his/her own desired walking speed. It is influenced by many factors such as intention outlook, age, and gender [13, 28, 29, 32]. We have used Gaussian distribution model, which offers a normal distribution pattern similar to real-world scenarios.

Agents sometimes walk in groups especially in our case study [23]. Agents within the same group tend to keep close to the other members of the group. Even if they are separated, they will try to reunite as soon as possible.

Thus, adding these four new forces (intention outlook, gender, walking speed, and group) into the social force model, will give each agent more characteristic, which makes their behaviour more unique. These result in a better crowd simulation since agents do not move in a systematic manner in the real world

$$
\begin{aligned}
\vec{f}_{\text {ZSFM }}= & \vec{f}_{\text {driving }}+\sum_{\alpha=1}^{n} \vec{f}_{\text {repulsion }}+\sum_{\alpha=1}^{n} \vec{f}_{\text {attraction }}+\sum_{\alpha=1}^{n} \vec{f}_{\text {goal }} \\
& +\sum_{\alpha=1}^{n} \vec{f}_{\text {gender }}+\sum_{\alpha=1}^{n} \vec{f}_{\text {speed }}+\sum_{\alpha=1}^{n} \vec{f}_{\text {group }}
\end{aligned}
$$

The first step to implement this model into simulation is to construct all the algorithms that will be used in this research. All the algorithms are based on Eq. 4. Each of the key algorithms will be explained in detail below.

\subsubsection{Intention outlook}

Intention outlook is significant in this model, without that pedestrian will not have their target and do not know which direction they want to take. Thus, each pedestrian will just stand still in their current position. Intention outlook reposition can be summarized as Algorithm 1 with the time complexity of $O(n)$, where $\mathrm{n}$ is the number of agents.

Algorithm 1 Intention Outlook reposition calculation

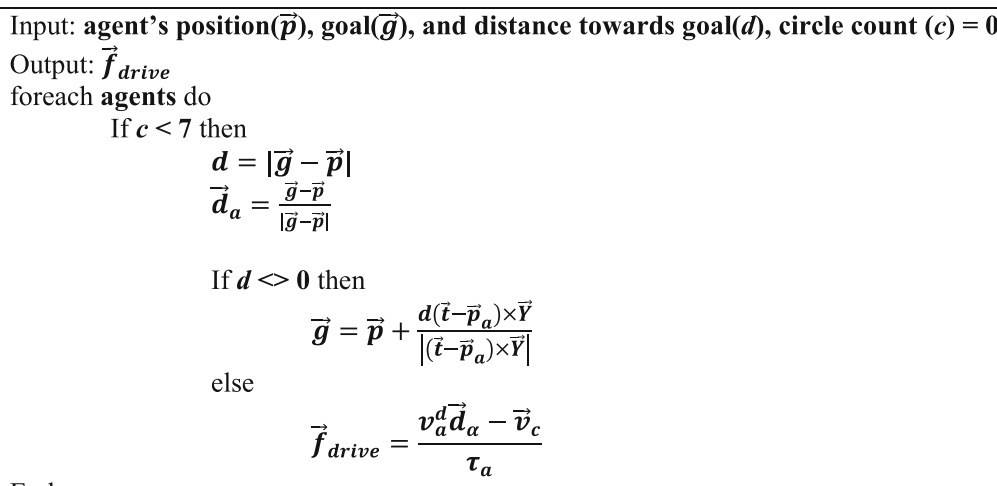




\subsubsection{Gender}

Pedestrians with different gender have greater interaction forces than pedestrians with the same gender, especially for Tawaf. To simulate this behaviour, each agent will be set with two different sets of strength $(f a, f b) . f a$ is for same gender interaction and $f b$ is for opposite gender interaction. Based on what we discussed already, the gender interaction can be summarized as Algorithm 2 with the time complexity of $O(n)$, where $\mathrm{n}$ is the number of agents.

\subsubsection{Grouping}

Grouping is also a common sight within high-density crowd. This is because most agents come together with their family and friends. The agents within the same group tend to keep close with each other. If they get separated, they will try to get back together as soon as possible. Therefore, this behaviour is very important to be integrated into the crowd simulation model.

Algorithm 2 Gender repulsion strength calculation



\subsubsection{Walking speed}

Each pedestrian has its own walking speed depending on their gender, age and other factors which are summarized in Algorithm 3 with the time complexity of $O(n)$, in which $\mathrm{n}$ is the number of agents.

Algorithm 3 Walking speed distribution

Input: $\overrightarrow{\boldsymbol{v}}_{\boldsymbol{\alpha}}, \boldsymbol{d}_{\boldsymbol{\alpha}}, \overrightarrow{\boldsymbol{v}}_{\boldsymbol{c}}, \boldsymbol{\tau}_{\boldsymbol{a}}$
Output: $\overrightarrow{\boldsymbol{f}}_{\text {drive }}$
foreach agents do
\[ \begin{aligned} \overrightarrow{\boldsymbol{v}}_{\alpha}=\text { Gaussian distribution function } \\$\[ \overrightarrow{\boldsymbol{f}}_{\text {drive }}=\frac{\boldsymbol{v}_{\boldsymbol{a}}^{\boldsymbol{d}_{\boldsymbol{\alpha}}}-\overrightarrow{\boldsymbol{v}}_{\boldsymbol{c}}}{\boldsymbol{\tau}_{\boldsymbol{a}}} \]$ \\ \text { End }\end{aligned} \]

The grouping force will follow two of Reynolds rulebased models. The rules are cohesion and alignment. Algorithm 4 controls this characteristic.

Cohesion, $\overrightarrow{\boldsymbol{c}}_{a}$, is for the formation of the group (Eq. 5). Each agent location $\overrightarrow{\boldsymbol{p}}_{a}$ within the same group will be summed together and then divided by the number of the group members, $m$. Hence, the groups will move together to the same position.

$\overrightarrow{\boldsymbol{c}}_{a}=\frac{\sum \overrightarrow{\boldsymbol{p}}_{a}}{m}$

The next rule is alignment, $\overrightarrow{\boldsymbol{m}}_{a}$, which is used to make sure that every group member moves at the same walking speed (Eq. 6). A group cannot have a member who walks too fast or too slow. They must have similar speeds to move together. Hence, the average speed of the group will be calculated. The total walking velocity of each agent, $\vec{v}_{a}$, 
will be divided by the total number of the group members, $m$ :

$\overrightarrow{\boldsymbol{m}}_{a}=\frac{\sum \vec{v}_{a}}{m}$

The separation rules are eliminated because the repulsion force of the social force model has a better solution for collision detection compared to this rule-based model. In addition, if the separation rule is implemented on top of the social force model, it will cause redundant of repulsion forces. This will make the agent move awkwardly and unnaturally.

As a conclusion, the grouping force will consist of two rules of Reynolds rule-based models. These rules are cohesion and alignment. These two rules will be integrated with the rest of the ZSFM to make a better crowd simulation model (Eq. 7).

$\sum_{\alpha=1}^{n} \vec{f}_{\text {repulsion }}=\overrightarrow{\boldsymbol{c}}_{a}+\overrightarrow{\boldsymbol{m}}_{a}$

The time complexity of Algorithm 4 by considering $n$ agents and an average number of $m$ people in the groups is $O(n+m)$. Due to the fact that $m$ is quite smaller than $\mathrm{n}$, the whole time complexity is $O(n)$.

Algorithm 4 Grouping calculation

$$
\begin{aligned}
& \text { Input: } \overrightarrow{\boldsymbol{p}}, \overrightarrow{\boldsymbol{v}}, \text { group }, \overrightarrow{\boldsymbol{m}}=\widehat{\mathbf{0}} \\
& \text { Output: } \overrightarrow{\boldsymbol{f}}_{\text {group }} \\
& \text { foreach agents do } \\
& \text { If agents } \\
& \qquad \begin{array}{r}
\overrightarrow{\boldsymbol{p}}=\frac{\sum \overrightarrow{\boldsymbol{p}}}{\boldsymbol{m}} \\
\overrightarrow{\boldsymbol{v}}
\end{array}=\frac{\sum \overrightarrow{\boldsymbol{v}}}{\boldsymbol{m}} \\
& \boldsymbol{m}++
\end{aligned}
$$

End

\subsection{Crowd control model}

This section discusses the stopping motion of the highdensity crowd simulation. Most current existing model only focuses on the walking motion of the crowd simulation; thus, it cannot be used to handle these types of situations. This study suggested a solution by introducing two new stopping motions into the high-density crowd simulation, which are sudden stopping and slow stopping.

\subsection{Motion stopper}

In crowd simulation, it is not enough just to simulate the path of each agent, and it is also important to calculate the motion of each agent. Most researchers focus on the simulation of crowd walking motion. However, agents do not just walk forever until they reach their destination. Sometimes they stop for some reason such as talking to other agents or seeing something that grabs their attention $[7,19,34]$. Stopping motion in high-density crowd simulation is in need and is presented in this section using a heuristic method for two new stopping motions. First is sudden, which happens once an agent stops abruptly along the way his/her path. This is because the agent suddenly encounters huge conflicting forces. As an example, when another agent suddenly crosses in front of the walking agent. The second stop motion is the slow stops, which happens if the agent slows down before completely stop walking. This is because the agent encounters small but continuous conflicting forces. For example, when the agent follows groups of slow-moving agents, both scenarios will cause pedestrians to stop if the conflict is big enough to make the moving forces closer to zero. Agents will stop in their position for a while before start moving again. It is because the pedestrians will need to gain enough momentum to start moving [5, 51].

\subsubsection{Fundamental of motion stopper}

This research has developed another novel model called motion stopper model (MSM). MSM is a new model used to control the stopping motion of agents. This model also created new mathematical equations to make it suitable for high-density crowd scenarios. This model was based on the agents' walking speed. MSM consisted of two main motions: stopping and walking (Fig. 6(left)). This is because sometimes agents are walking and sometimes the agents are stopping. The current crowd simulation model treats both of this behaviour mutual exclusive.

\subsubsection{Motion stopper model (MSM)}

Motion stopper model (MSM) is used to simulate the stopping motion of each agent. MSM basically has two states, which is walking state and stopping state. The transitions between these two states are controlled by the velocity of each agent (Fig. 6 (right)). Agents who are in the walking state will change to stopping state if their velocity decreases to almost zero or lower. On the other hand, agents who are in stopping state will start walking again if their velocity increases above zero. The increasing and decreasing velocity is influenced by the conflicting force towards the agents. As has been already explained, if the agents encounter huge conflicting forces towards them, it will force them to stop walking unexpectedly. 

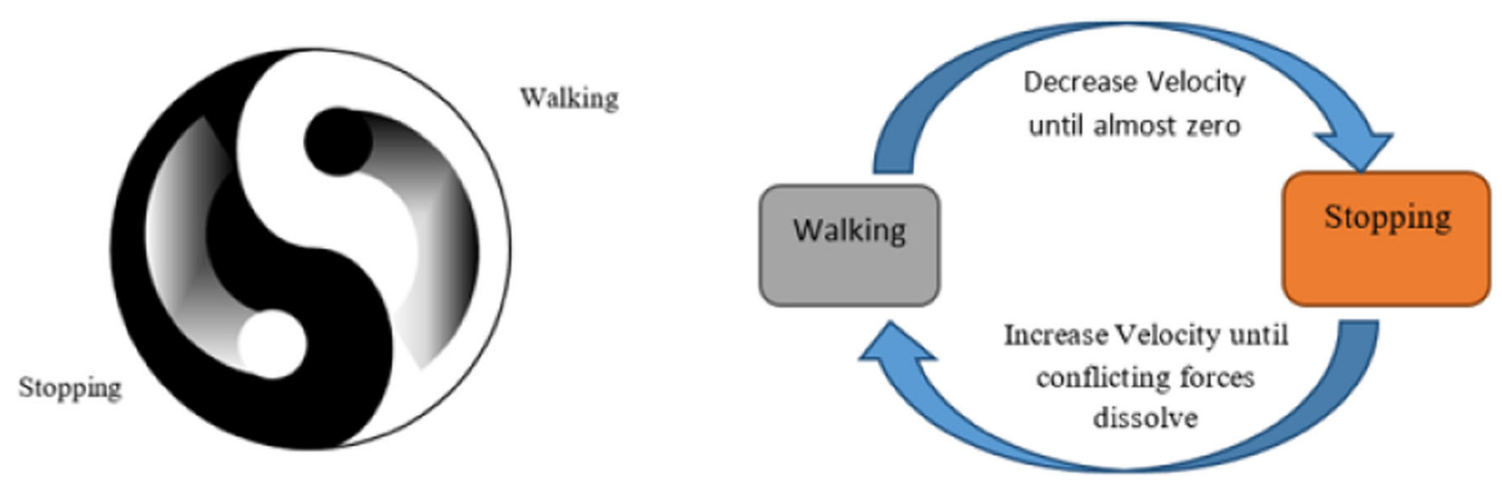

Fig. 6 (Left) Motion stopper model, (right) relationship between stopping and walking

2.3.2.1 Stopping motion MSM controls the stopping motion of every agent. If the velocity of the agent is almost zero, the motion of that agent will stop too. It is to avoid the agents walking in the same place which are unnatural. Other than that, MSM also controls when the agent will start walking again. A stopping agent cannot start walking again immediately after stopping because the agent needs to regain its momentum to start walking again $[5,51]$. Thus, the agent needs to halt for a few seconds before start walking again. This is done by manipulating the velocity of the agent as seen in the equations above. If the agents do not reach a certain value of velocity threshold, he or she will remain static until the velocity value is over the threshold.

$\vec{f}_{\text {stopper }}= \begin{cases}0 & \left|\vec{f}_{\text {walk }}\right|>0.01 \\ 0.001, & 0.001<\left|\vec{f}_{\text {walk }}\right| \leq 0.01 \\ \vec{f}_{\text {walk }}, & \left|\vec{f}_{\text {walk }}\right| \leq 0.001\end{cases}$

Equation 8 explains about how the MSM will be introduced into the crowd simulation. MSM will initiate a conflicting force $\vec{f}_{\text {stopper }}$ towards the agent walking force $\vec{f}_{\text {walk }}$ if it is less than the slow stopping threshold value which is 0.01 . This will cause the agent to have a slow stopping motion. It is because the moving force is quite low to walking normally. Thus, the agent walking force will gradually decrease based on time. However, if the walking force is below the sudden stopping threshold (0.001), the agent will stop walking abruptly, which is the sudden stopping motion. The walking force $\vec{f}_{\text {walk }}$ of the agent is too low to walk. In the simulation, the agent would appear like its vibrating. Thus, by introducing the stopping motion, this vibrating agent is eliminated, which makes the agent stop moving.

Since the algorithm (Algorithm 5) is based on the mathematical equation above, the explanation is quite the same. The time complexity of Algorithm 5 for each agent is $O(1)$, while for n number of agents is $O(n)$. The agent will have a slow stopping motion if the moving force is less than 0.01 and sudden stopping motion if the moving force is less than 0.001 . The agent will start walking again if the agent's moving force is higher than the threshold, which is 0.01 .

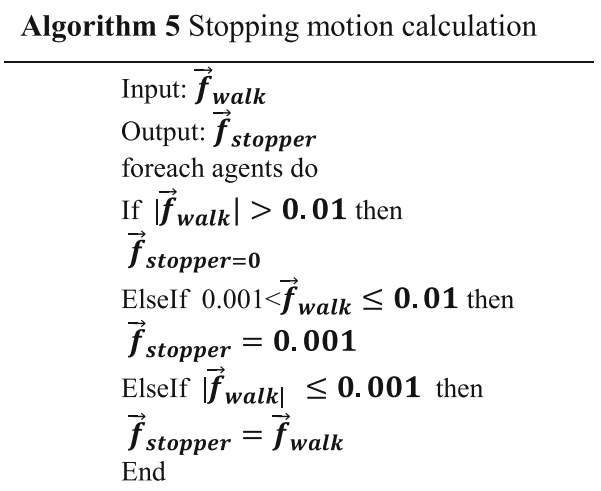

\subsubsection{Walking motion}

After stopping, the agent cannot suddenly start walking again. The agent needs to wait for a few seconds to gain walking force $\vec{f}_{\text {walk }}$ to start walking again. If the agent starts walking again too quickly, it is not natural because a human can start walking so fast so abruptly unless they are in a sprinting race (Eq. 9):

$\vec{f}_{\text {walk }}= \begin{cases}0 & \left|\vec{f}_{\text {walk }}\right|<0.01 \\ 0, & \text { time }<2 \text { seconds } \\ \vec{f}_{\text {walk }}, & \left|\vec{f}_{\text {walk }}\right| \geq 0.01\end{cases}$

Therefore, each agent will be given a two-second buffering time before they can start walking again. After two seconds of time, if the agent gains enough walking force $\vec{f}_{\text {walk }}$ (above the walking threshold, 0.01), the agent will be animated. If the force $\vec{f}_{\text {walk }}$ is too low (below the walking threshold, 0.01), the agent will remain in the stopping phase until they gain enough walking force. 


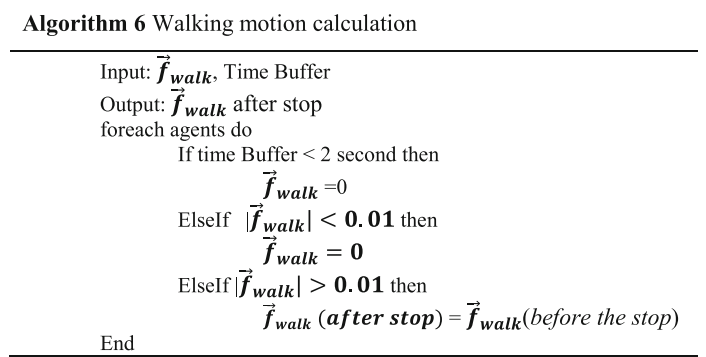

The walking threshold value (0.01) was based on the experiment which is done by making a stopping agent to slowly gain enough walking force. The experiment is repeated until the optimal walking threshold is found. Each agent in the experiment is given a range of walking force (0.1-0.0001). The optimal walking threshold is 0.01 which shows the best walking motion compared to the other value.

A similar experiment is conducted for the walking time buffer period. For the starting of the experiment, each agent is in the walking state. In the middle of their path, the agent was given the same conflicting forces which make them stop. After that, each agent will be given the same walking forces to make them start walking again. The only difference is that each of them is given a different walking time buffer, ranging from 0.5 to $5 \mathrm{~s}$. Based on the experiment results, it shows that the optimal walking time buffer is $2 \mathrm{~s}$, which shows a natural stop and walking again motion.

Since the algorithm (Algorithm 6) is based on the mathematical equation above, the explanation is quite the same. The time complexity of Algorithm 6 for each agent is $O(1)$, while for n number of agents is $O(n)$. The agent will start walking again if the agent passes the time buffer. After that time buffer, if the agent's force is high enough, they will start moving again. If not, they will stay in the stopping phase until they gain enough force.

\section{Integration and framework}

This section discusses the integration between the Zahmah social force model (ZSFM) and the motion stopper model (MSM). The integration will produce a novel crowd simulation framework that improves the realism of the highdensity crowd simulation.

\subsection{Architecture of high-density crowd simulation}

This section explains the process of designing and creating 3D models of Kaabah and pilgrims (agents) using MeshLab tools. The models are created using 3D max and viewed by using MeshLab tools. Later, the models are saved into the Collada model which is supported by the horde 3D game engine.

The high-density crowd simulation is divided into three main elements: input, main process, and output. The input of the simulation is the 3D models of agents and Kaabah. The main process of the simulation is the rendering of the crowd, which consisted of two main parts: ZSFM and MSM. The ZSFM will control the movement of the agents, while the MSM will control the motion of the agents. The output of the system is the high-density crowd simulation (Fig. 7).

The rendering process is started by interpreting the 3D Collada model and then arranging all those models into a proper position. The first object rendered in this application is the human character model (agents). The reason being is the agents have a huge number of polygons compared to the Kaabah model. After the rendering process of the agents, it is continued with the Kaabah rendering. The agents are placed in a certain location around the Kaabah. The movement and motion are control by ZSFM. The output of the system is a mass of high-density crowd circumambulating the Kaabah as the epicentre.

\subsection{High-density crowd simulation framework}

In simulating the high-density crowd simulation, it is not enough just to simulate the movement of the crowd. It is also important to simulate the motion and animation of the crowd, especially if the simulation is in three dimensions (3D). The motion part plays an important role in capturing the realism of the high-density crowd simulation. An awkward and unnatural agent's motion within the crowd will make the simulation less realistic.

Thus, this research proposed two novel models: the ZSFM and the MSM which control the crowd movement and crowd motion, respectively. Later, these models were integrated together into a new crowd simulation framework to produce a more realistic high-density crowd simulation. The integration will control every aspect of the crowd behaviour microscopically.

The proposed framework combines the parameters (gender, walking speed, intention outlook, and grouping) of the crowd simulation model. The time complexity of the proposed model for each agent is the cumulative complexity of all presented algorithms with the proposed model in Eq. 4, which remains as $\sum_{1}^{n}(6 * O(n))=O\left(n^{2}\right)$. Therefore, memory consumption is not a concern in this method. As an example, here is the memory usage of Algorithm 1: $3 * 4+3 * 4+8+4+3 * 4+3 * 4+$ $3 * 4+3 * 4+8+3 * 4+8=48+112$ Byte. Thus, the 
Fig. 7 Architecture of highdensity crowd simulation

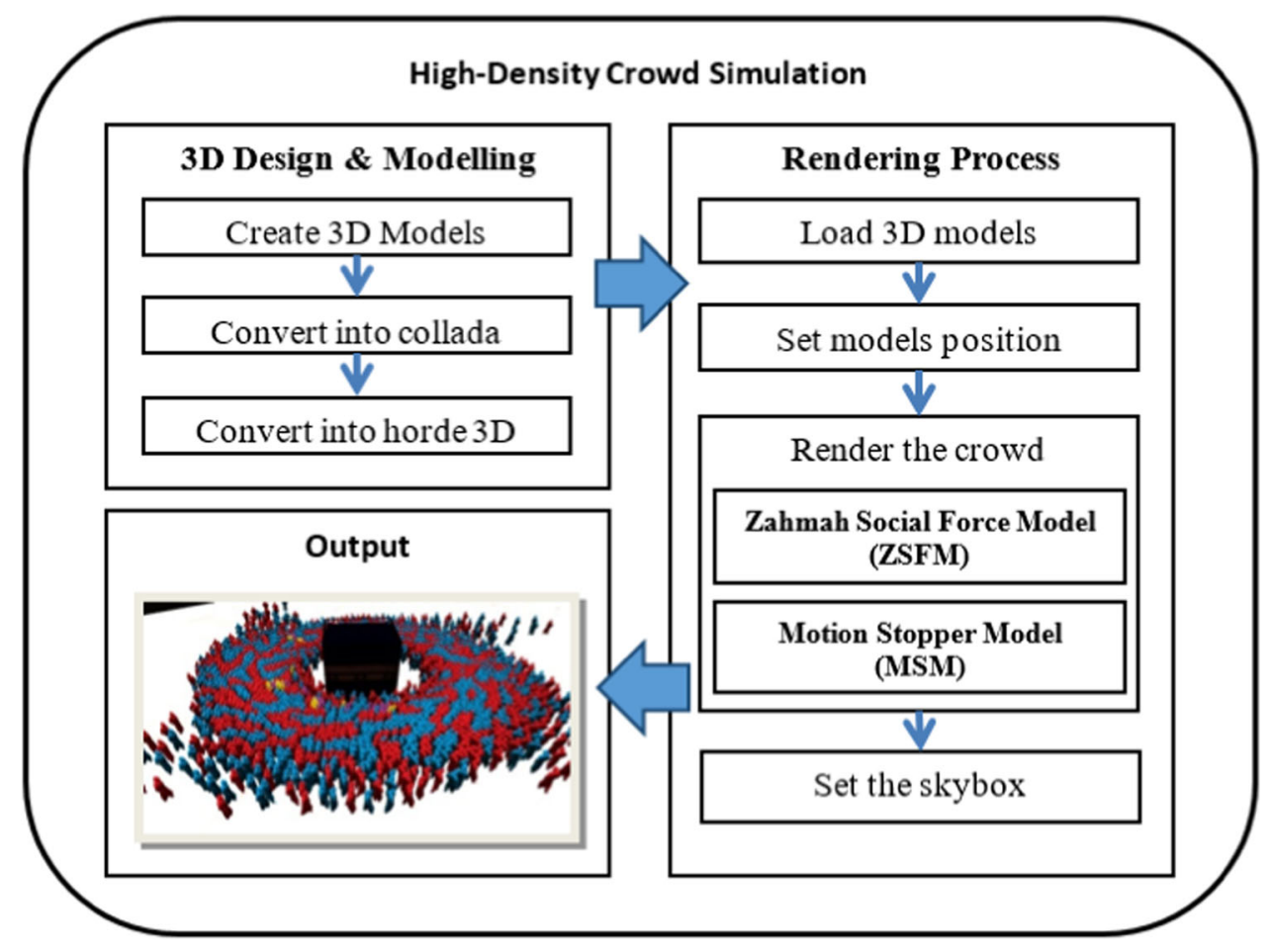

contribution of this algorithm in the proposed model is $112 * n$ Byte which is still less than $\mathrm{O}\left(\mathrm{n}^{2}\right)$.

This is to enable the simulation of a high-density crowd as realistically possible. This is represented in Fig. 8. Agents in this model will interact with other agents in order that some grouping can be managed. The reasoning for this is that many of the agents are grouped via family or friends.

The integration is crucial to make the high-density crowd simulation as realistic as the real-world situation. Thus, the integration is being done firstly by implementing the crowd simulation model into the high-density crowd. This model controls the movement and behaviour (walking



Fig. 8 The overview on the integrated model speed, gender, and intention outlook) of all agents within the crowd simulation. After that, the crowd control model will be added to the crowd simulation model. The crowd control model controls the stopping behaviour of the agents. Thus, it makes the crowd control model as the opposite of the crowd simulation model.

Microscopically means that each agent within the highdensity crowd simulation will have their own identity, characteristic, and motion (Fig. 9). Each agent will be given a random intention outlook, gender, walking speed, and groups based on the ZSFM. Later, during the simulation, the agent motion whether stopping or walking is controlled by the MSM. All the behaviour done by the agents is fully autonomous.

\section{Results and evaluation}

In the final evaluation experiments, both models ZSFM and MSM are combined to complete the high-density population crowd framework. This high-density crowd simulation will have all the new forces introduced in the ZSFM (intention outlook, gender, walking speed, and grouping) and also both stopping motion of the MSM (sudden stop and slow stop). The population for these experiments was set to be 45,000 agents. The Tawaf ritual was used as the case study for these experiments.

The simulation has been performed in Visual Studio over core $\mathrm{i} 7$ with 3.x GH speed and $8 \mathrm{~GB}$ of memory 


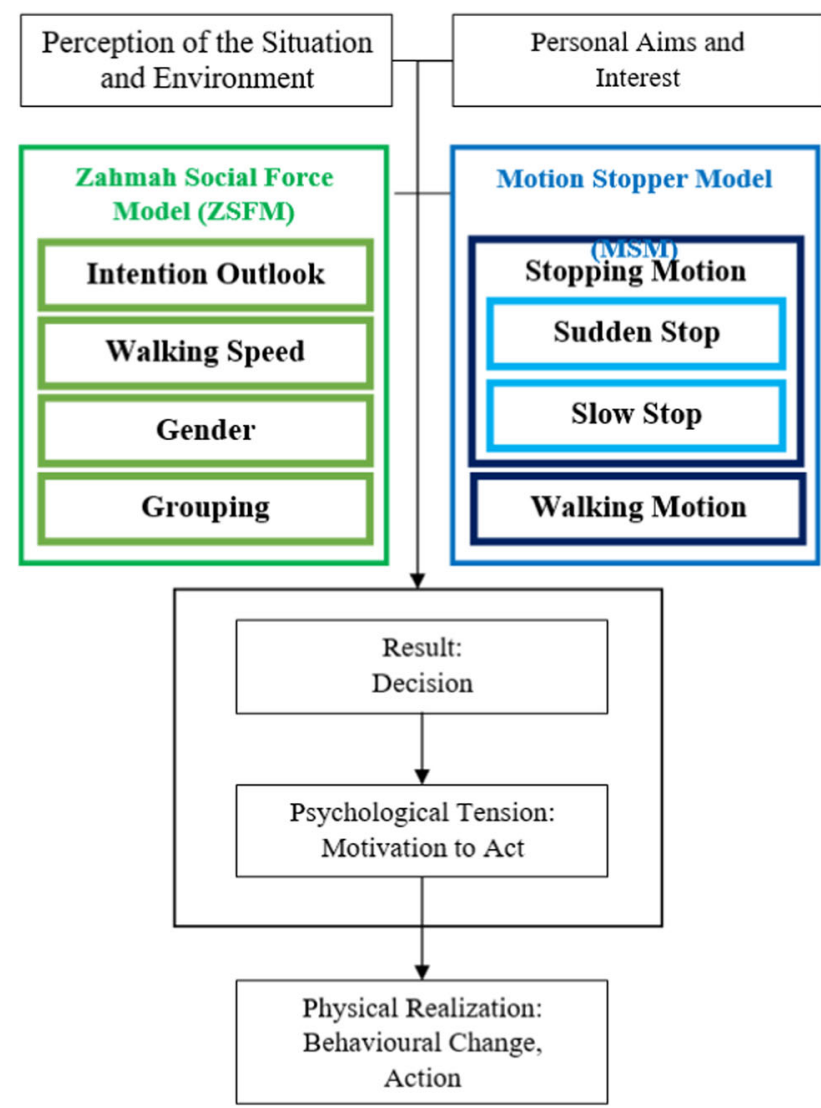

Fig. 9 Details on high-density crowd simulation framework

running over Windows 10. Data were collected from Tawaf videos available online.

\subsection{Zahmah social force model}

To evaluate this characteristic, a simple experiment has been done. This experiment uses both social force model and ZSFM. However, in ZSFM only the walking speed force is integrated, while the other additional forces are not, to highlight the walking speed results. The population used for this test was 45,000 agents for both models. The test result (Fig. 10) shows that the walking speeds different in ZSFM will cause the agents to move more randomly and more scattered. Different from the social force model causes the agents to move in the uniform pattern since they are walking at the same speed. This proved that the walking speed distribution in ZSFM improves the realism of the high-density crowd simulation since in the realworld scenario, the agents would not walking in a systematic manner. Although the agents were in blue to represent male agents and red to represent female agents, it does not represent anything significant yet at this level (Fig. 10).

\subsubsection{Intention outlook comparison}

To test the intention outlook forces within the crowd simulation, the experimental setup will be using the same approaches with the previous experiment. The intention outlook forces will be integrated into the crowd simulation, while the other new forces are not, to emphasize the effect of intention outlook in the crowd simulation. This experiment will use 4500 agents' populations for both ZSFM and social force model comparison. Based on the experiment result, it shows that the ZFSM crowd flows are more hectic and unsystematic, compared to the Social Force Model. This is because the agents have their own desired intention outlook, which means their own preferred distance towards the epicentre. Unlike the agents in the social force model which all have the same intention outlook, which causes them to move systematically and organized, here they are more realistic in the such movements. This proved that integrating the intention outlook into the high-density crowd simulation will improve the simulation result (Fig. 11).

\subsubsection{Walking speed comparison}

The first test is the walking speed distribution test between both conventional social force model and the proposed model, ZSFM. The walking speed for each agent in the ZSFM was distributed by using the Gaussian model. Meanwhile, the walking speed for each agent in the social force model was distributed evenly by calculating the average walking speed of each agent. In real-world situations, it is impossible to assume that every agent will be walking at the same speed. Thus, this research proposed the Gaussian model to distribute the walking speed for each agent (Fig. 12). By using the Gaussian model distribution, it is most likely to be more realistic to the real-world situation [39] because it will distribute the walking speed more naturally. Some of the agents will be walking slower and some agents will be walking faster.

\subsubsection{Gender interaction comparison}

The second evaluation is the gender interaction force experiment for both social force model and ZSFM. The gender interaction force was applied because of the different respect of personal space between the two genders [4]. Women have more respect in terms of personal space; hence, they will have a stronger interaction force compared to men. Hence, in ZSFM there are two different interaction forces for every agent. The first interaction force was between an agent with the same gender (men with men and women with women), and the second interaction force is between two different genders (men with women). The 
Fig. 10 Walking speed simulation using social force model, (bottom) crowd simulation using ZSFM (blue = male agent, red $=$ female agent $)$ comparison result: (top) crowd


Fig. 11 Intention outlook force comparison results: a crowd simulation using social force model; $\mathbf{b}$ crowd simulation using ZSFM (blue = male agent, red $=$ female agent)


latter interaction will have a stronger force than the former interaction as shown in Figs. 13 and 14. Although the interaction forces are different for both interactions, the interaction threshold (interaction distance) stayed the same. The reason is that the personal space of the agent is considered the same for every agent. Based on the same figure, it shows that the social force model only has one interaction force. It is because previous researches do not consider gender differences as an important factor to be considered in the high-density crowd simulation.

Similar to the previous experiment, this experiment also will use the same test case scenario which is the Tawaf ritual and also the same 4500 agents' population. The experiment compared the simulation results between the social force model and the ZSFM. In this test, only the gender interaction force will be integrated, while the other new forces within ZSFM will not, to emphasize the result 




Fig. 12 Walking speed distribution of both ZS force model and social force model

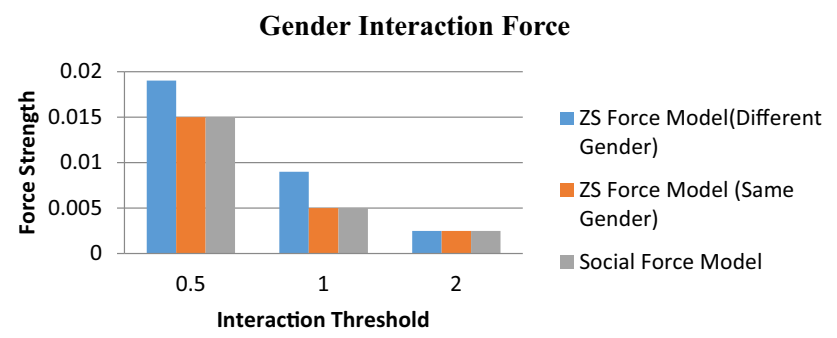

Fig. 13 Gender interaction forces of both ZS force model and social force model

of gender interaction force. The experiment result (Fig. 14) shows that the gender interaction forces in ZSFM have a clear distinction between both genders (men in blue and women in red). This is due to the interaction forces differences between both genders. The agent will have a greater repulsion force towards the opposite gender agents. Consequently, it will make the agents with the same gender walk closely together. Compared to the conventional Social Force Model, all agents seem to be walking equidistance from each other regardless of gender. Thus, it shows that the ZSFM improved the gender interaction behaviour of the crowd since both genders will have a different interaction with each other.

\subsubsection{Grouping comparison}

Next is the grouping force test. Some agents within a crowd prefer to be in a group. Each group consists of two or more agents depending on the size of the group. These groups will walk closely together and will regroup back if they are separated whenever possible. Hence, in order to demonstrate this situation in the simulation, several groups had been created within this simulation. These groups were generated manually because to have a controlled grouping environment so that the results are comparable between crowd simulation with grouping forces and simulation without it. The agents within groups are in yellow coloured, while the other agents without groups are in either red or blue depending on their gender. There are no distinction colours of gender in groups because there are no gender interaction forces between agents within the same group. This is because the agents from the same group would have the same interaction force strength for both genders.

The experiments were run by using 4500 agents as the total population for both social force model and ZSFM. Similar to the previous tests in order to emphasize the
Fig. 14 Gender interaction force comparison results: (top) crowd simulation using social force model; (bottom) crowd simulation using ZSFM (blue $=$ male agent, red $=$ female agent
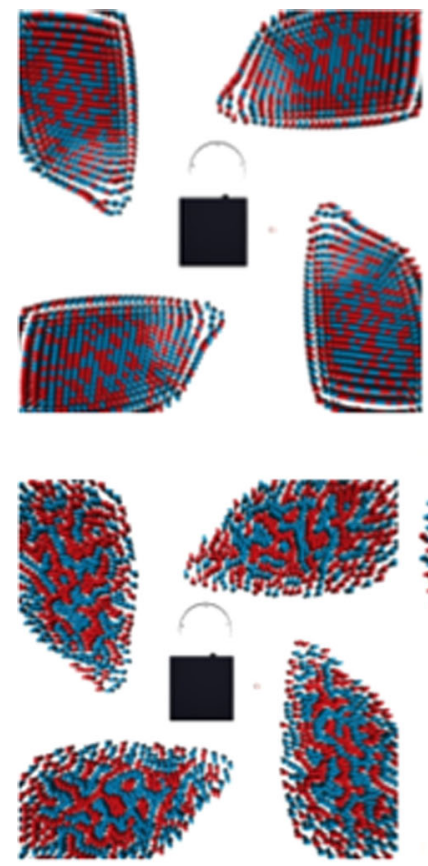
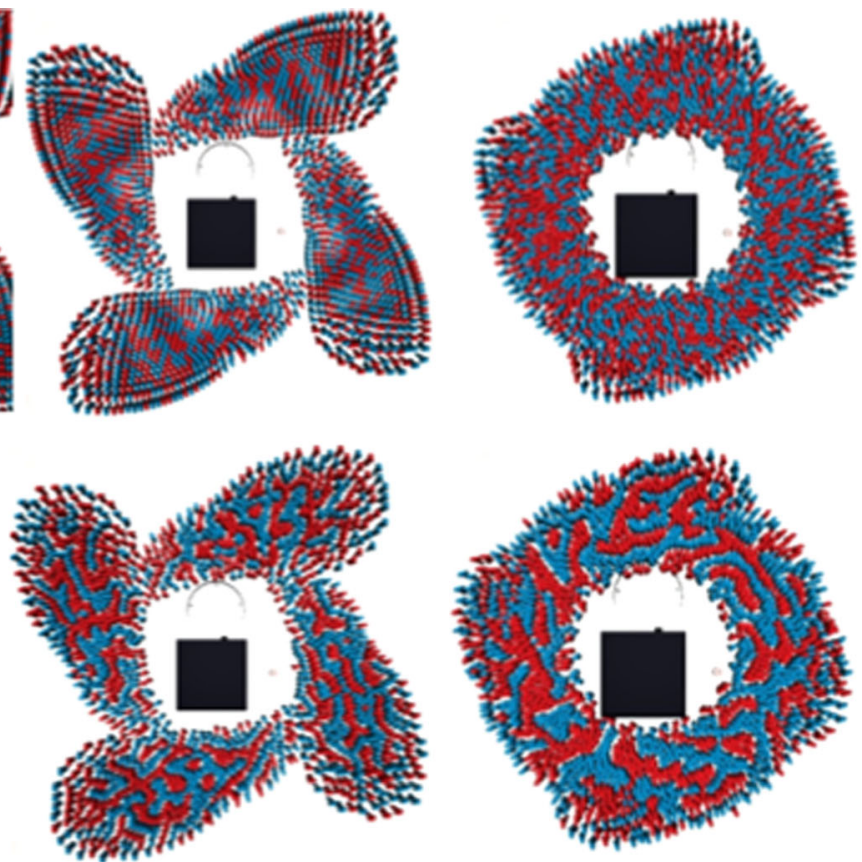
grouping force effect, only the grouping force was integrated, while the other additional forces of ZSFM are not. Figure 15 shows the simulation results for both social force model and ZSFM in the Tawaf scenario. It shows that the grouping force in ZSFM will make the agent from the same group to stay together even though the agents were in a congested area. Therefore, we can see clearly that those agents were from the same group. This is different from the agents in the social force model which do not stay together and move individually. So, it does not show that the agent was in the same group. With the integration of grouping forces within the crowd simulation model, it improves the realism of the simulation because in the real-world scenario, agents within the same group will stick together. Even though they are separated, they will try to get back together as soon as possible, as shown in the ZSFM simulation result.

\subsubsection{Comparison with conventional social force model}

This experiment is to evaluate the simulation result of the combination of all new forces within the ZSFM which is intention outlook, walking speed, gender, and grouping. The result of the ZSFM will be compared to the result of the conventional Social Force Model. The testing environment of this experiment will be the same for both models. Blue agents will represent the male agents, red agents will represent the female agents, and yellow agents will represent the agents who are in groups.

The experiment results show the combination of all the forces within ZSFM made the agents move more randomly and more hectic, compared to the social force model (Fig. 16). This is similar to the behaviour of the highdensity crowd in the real-world scenario. The conventional social force model made the agents move in a uniform pattern like companies of soldiers marching around the Kaabah. This behaviour was unrealistic, and it does not happen in the real-world situation. Thus, it proved that the ZSFM is better in simulating a high-density crowd compared to the Social Force Model.

\subsection{Motion stopper model}

\subsubsection{Comparison with social force model}

The experiments will be using social force model for controlling the high-density crowd behaviour, as MSM cannot standalone without any crowd simulation model. Since MSM is a new model that focuses on stopping motion in a congested area, there is no other suitable model to be compared. Therefore, the experiment will show the results of high-density crowd simulation by integrating MSM against without integrating MSM. The experiments are run using the same case study which Tawaf for consistencies of the results were set.

The other stopping motion models require a door or wall to occur, while MSM does not require any obstacle other than the density of the crowd to occur. This thesis will compare the social force model without integrating MSM and with integrating MSM. This comparison is to prove whether integrating the MSM improved the realism of the simulation or not. Both bottlenecks effect and arc
Fig. 15 Grouping comparison result a crowd simulation using social force model; $\mathbf{b}$ crowd simulation using $\mathrm{ZS}$ force model (blue $=$ male agent, red $=$ female agent, yellow $=$ agent in groups)
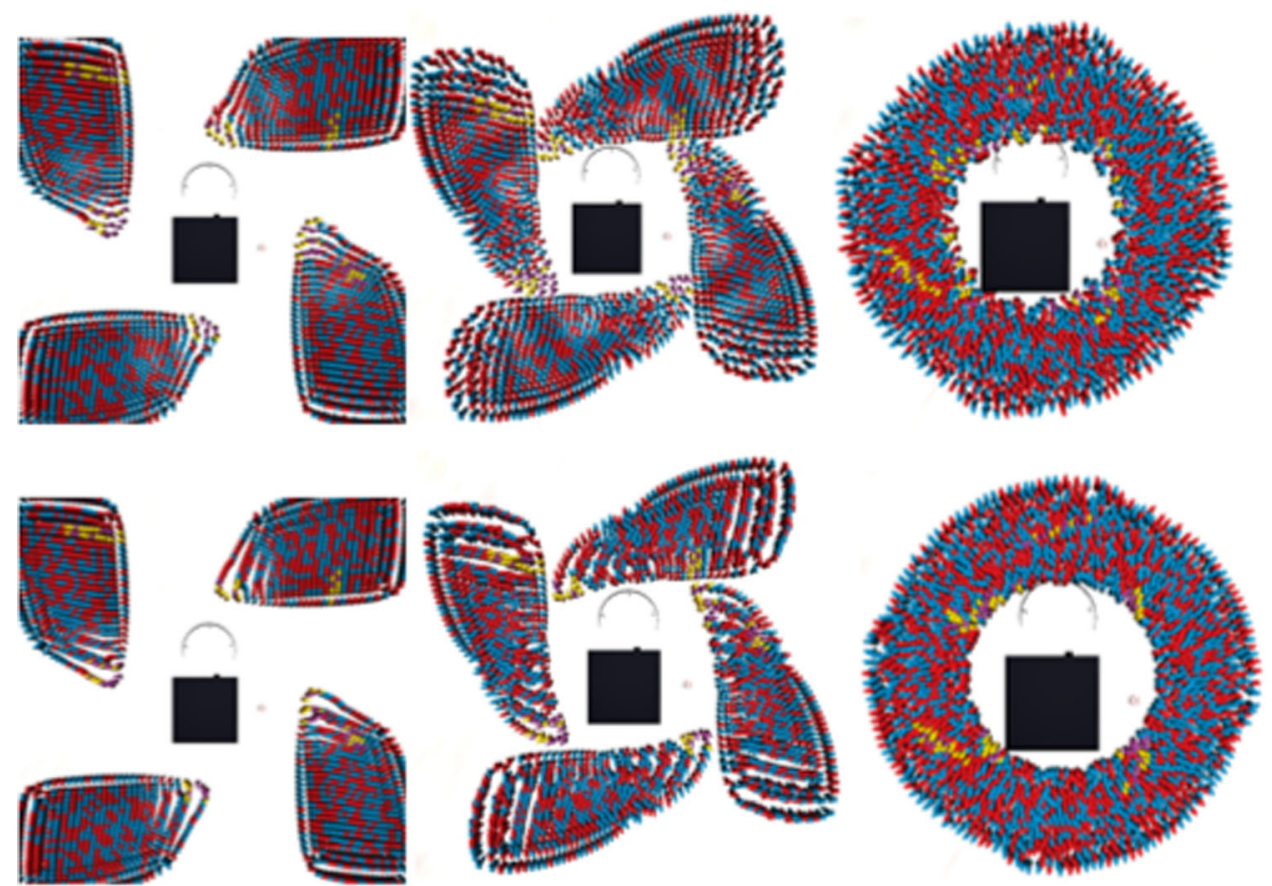
Fig. 16 Social force model and ZSFM comparison results: (top) crowd simulation using social force model, (bottom) crowd simulation using ZSFM (blue = male agents, red $=$ female agents, yellow $=$ agents in groups)
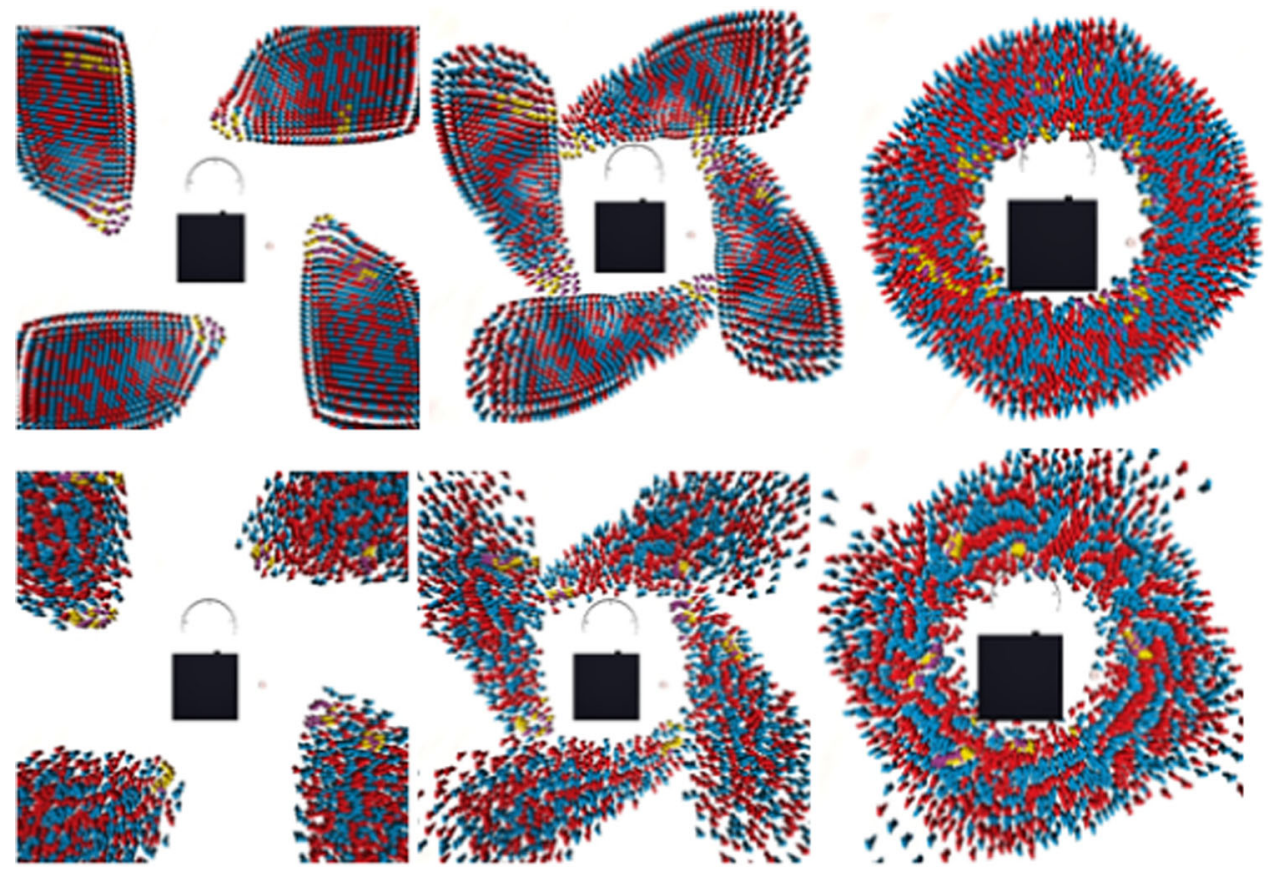

formation require a specific environment condition that makes the stopping behaviour to happen.

Figure 17 (top) shows the bottleneck effect of stopping behaviours. The stopping behaviour requires a narrow pathway for it to happen. The crowd flow is also bidirectional. Thus, it is totally different scenarios with this thesis scenario which is Tawaf which does not need a wall and the crowd flow is only in one direction to make the stopping behaviour to happen.

Although the arc formation is also a one direction crowd flow simulation, it requires a door to make the stopping behaviour to occur (Fig. 17 (bottom)). Since the Tawaf
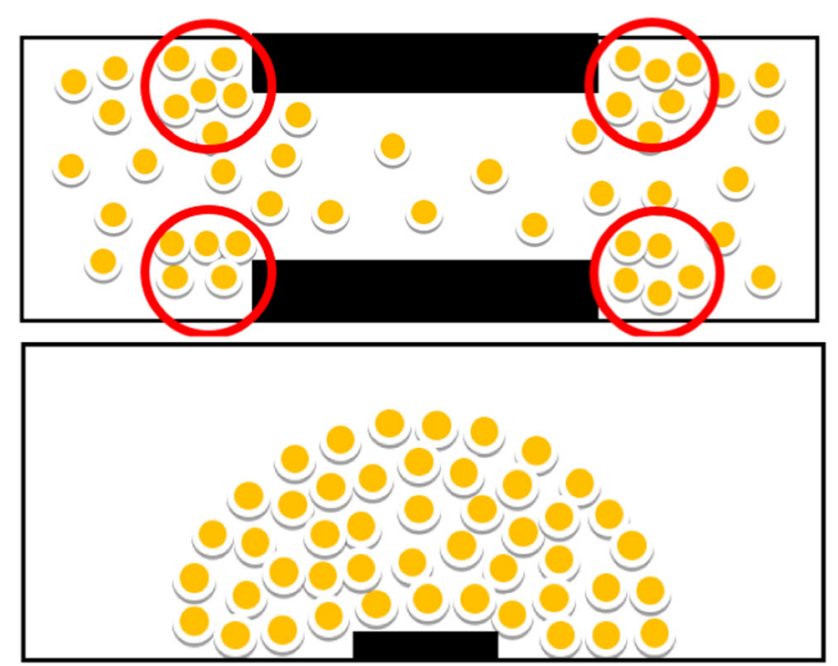

Exit

Fig. 17 (Top) Bottleneck effect, (bottom) arc formation ritual does not have any doors for the agents to get through, it is not suitable to be compared to the arc formation stopping model. Other than that, mostly during the evaluation of the arc formation, it requires a panic situation to make the arc to form clearly. This research does not simulate any panic situation only in a calm and normal situation.

As we can see in Fig. 18, it shows that by implementing the MSM into the high-density crowd simulation, the stopping motion was handled much better compared to the Social Force Model. Without the MSM, the stopping will not halt for a few seconds before continue walking again. As a result, the agent will change from walking to stop very abruptly. This is a contrast from with MSM, in which each stopping agent will halt for a few seconds before start walking again. This is a more natural behaviour because pedestrians [41] in the real world must regain their momentum before they walk again after stopping. Besides, the MSM has two different stopping motions: sudden stop and slow stop. These stopping motions were represented as red agent for sudden stop and orange agent for slow stopping. Both motions improved realism of the simulation result by eliminating agent who walks in the same position, which is not natural. Other than that, the agent also will start walking more naturally with MSM compared without MSM, which starts walking abruptly without halting for a few seconds before continue walking again. In the realworld scenarios, the agent will halt for a few seconds after stopping to gain enough momentum before start walking again. 
Fig. 18 Social force model and MSM comparison results: a crowd simulation using social force model without MSM; b crowd simulation using social force model with MSM $($ red $=$ sudden stop, orange $=$ slow stop)
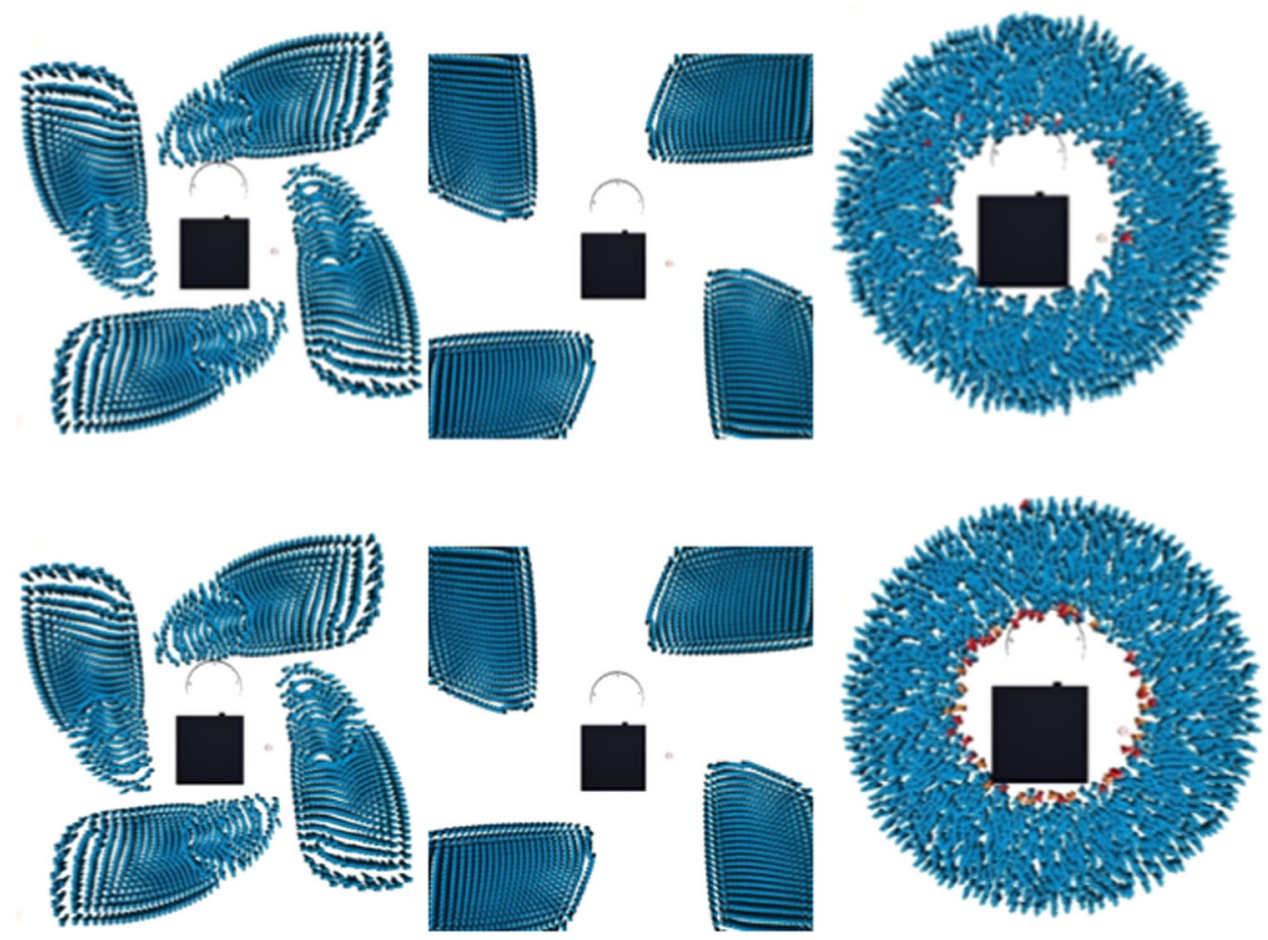

\subsubsection{Comparison with various population}

As stated before within this chapter, MSM is related closely with the number of agents within the simulation. These experiments use a different number of agents ranging from 500 agents to 4500 agents. The environment setup for all experiments was the same except the crowd population for consistencies of the results.

Figure 19 shows the increasing number of agents who stop either by sudden stop (red agents) or slow stop (orange agents) according to an increasing number of crowd population. Therefore, this test proves that the hypothesis was correct. The increasing number of population in the crowd will increase the stopping behaviour to happen more frequently. This is because the increasing population will also increase the density of the crowd, which directly will cause the agents, have less space between them to move around. It forced the agents to stop walking for a moment until they have enough space around them to start walking again.

The increasing numbers of stopping agents especially towards the centre of the circumambulation were mainly due to the high congestion towards the epicentre. Some stops occur at the outer ring of the circumambulation due to the agents cannot get into the inner circle because being blocked by the other agents. The occurrences of the slow stop were more common compared to the sudden stop because the agents were already walking slowly due to the congestion. Without the integration of MSM into the high- density crowd simulation, some of the agents will not stop moving around and sometimes even move at the same place due to low walking forces. By integrating MSM into the simulation, a movement like that can be eliminated because MSM will counter the walking forces with the opposite forces (stopping forces), which will make the agent stop moving before continues walking again. Thus, it will make the high-density crowd simulation more natural.

Figure 20 shows the stopping frequencies for $60 \mathrm{~s}$ of simulations based on the population from 500 agents to 4500 agents. There were no agents stops in 500 and 1000 populations because there were not enough agents to cause congestion in the simulation. The number of agents stopping increases as the population increases because the crowd becomes more congested especially towards the middle of the circumambulation. The increase in the congestion directly will increase the difficulty for the agent to walk, thus making the agents stop. The slow stop frequencies are higher than a sudden stop at the population of 1500 agents and then become lesser with the increase in the population. This is because the lesser number of agents means the less congested the crowd. So, the agents have an appropriate amount of space to slowly stop. On the other hand, in the more congested crowd, the agents do not have space and time to slowly stop which makes them stop abruptly. 
Fig. 19 MSM comparison

results by using different populations ranging from 500 agents to 4500 agents (red $=$ sudden stop, orange $=$ slow stop)


3000 Agents


Stopping occurrence frequencies

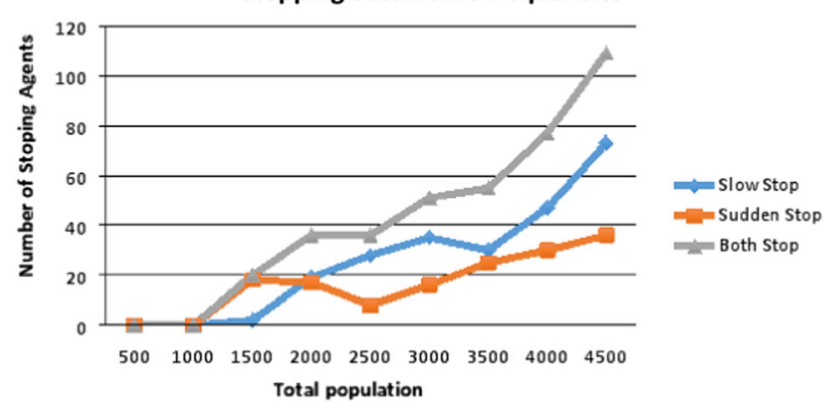

Fig. 20 Stopping occurrence frequencies based on the number of agents for $60 \mathrm{~s}$ of simulations

\section{Discussion}

These experimental results were compared to the realworld videos of the Tawaf ritual. The videos used in this test were taken from YouTube.

Figure 21 (top) shows the similarities between the simulation result and the real-world situation. Both were very congested especially towards the epicentre. At the outer layer of the circle of the simulation, the agents were walking sparsely and there was a lot of space between them, similar to the real-world scenario. This is because the agents in both simulation and real-world scenarios have their own preferred walking distance. Some of them prefer walking farther away from the epicentre for more comfort, 
Fig. 21 Comparing crowd simulation with real-world scenario, video of the Tawaf ritual [47]: (top: scenario 1) high-density crowd simulation compared to real-world scenario. (Middle: scenario 2) High-density crowd simulationgrouping; this is highlighted by white circles compared to realworld scenario video of the Tawaf ritual-grouping highlighted by yellow circles. (Bottom: scenario 3) Highdensity crowd simulation; orange agents demonstrate a slow stop and red agents demonstrate a sudden stop
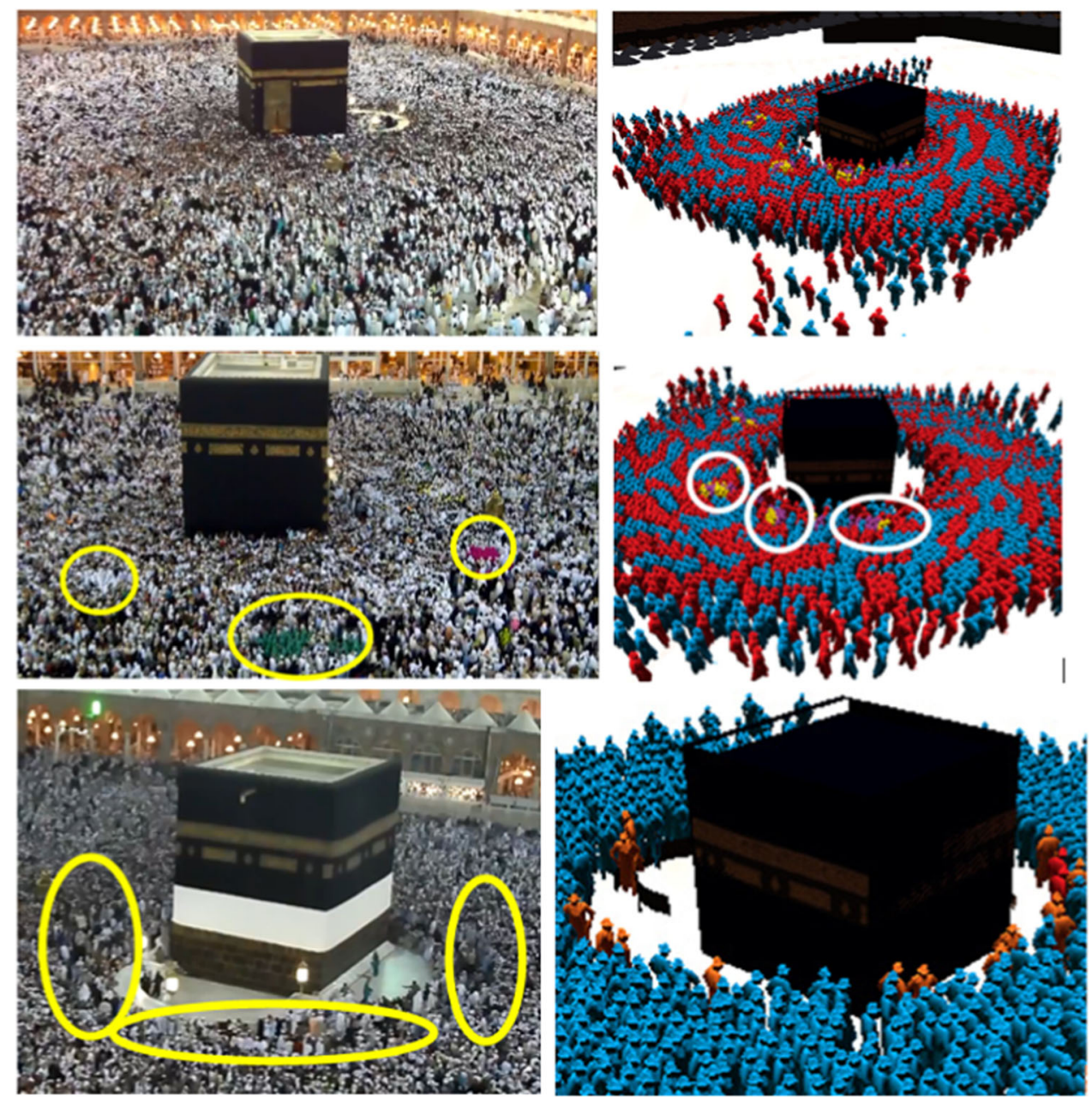

while some of them prefer walking closer to the epicentre because they want to get close to the Kaabah.

In the second video, it shows a very clear distinction between agents with groups and agents without groups (Fig. 21 (bottom)). It shows that agents within the yellow circle were from the same groups. They walk closely together for the entire length of the video. The same behaviour was also shown in the crowd simulation. The agents within the white circle also walk closely together while circling the Kaabah. This is due to the fact that some agents perform the rituals within groups. Agents within the same groups prefer to walk closely together to avoid getting lost in the crowd. Even if they get separated, they will try their best to get back together with their group. There is no distinction between the interactions with the opposite gender if the agents are from the same group because they know each other and some of them are their spouses, family members, or friends.

A detailed analysis of all the experimental results is provided to prove that this novel high-density crowd simulation framework is more efficient in simulating highdensity crowd compared to the Social Force Model. Based on all of the experimental results, the walking speed of each agent has been calculated. This is because the average walking speed of agents within the crowd simulation will be compared to the actual real-world data.

Average walking speeds were collected during previous experiments by using different populations (Table 3 ). The average walking speed $\overline{\boldsymbol{v}}$ was determined by using Eq. 10 . The sum of the walking speed $\boldsymbol{v}_{\alpha}$ of all agents within the simulation was divided by the population of the simulation, $m$. Based on the experiment results, it shows that the average walking speed during the real-world Tawaf situation $(0.3267 \mathrm{~m} / \mathrm{s})$ is quite near to the simulations average walking speed of the proposed high-density crowd simulation result for the population 45,000 $(0.35 \mathrm{~m} / \mathrm{s})$. This result shows that the simulation result average walking speed was comparable to the real-world situation which also has the population around 45,000 pilgrims [3].

$\overline{\boldsymbol{v}}=\frac{\sum \boldsymbol{v}_{\alpha}}{m}$

Average walking speed calculation example for the proposed framework (population of 500 agents): 
Table 3 Average walking speed of agents in the crowd simulations using the social force model and proposed framework for different populations

\begin{tabular}{lll}
\hline Crowd population & \multicolumn{2}{l}{ Average walking speed } \\
\cline { 2 - 3 } & Social force model $(\mathrm{m} / \mathrm{s})$ & Proposed framework $(\mathrm{m} / \mathrm{s})$ \\
\hline 500 & 2.00 & 1.58 \\
1000 & 1.90 & 1.55 \\
1500 & 1.83 & 1.24 \\
2000 & 1.20 & 0.83 \\
2500 & 1.10 & 0.82 \\
3000 & 1.05 & 0.62 \\
4000 & 0.99 & 0.58 \\
4500 & 0.93 & 0.54 \\
5000 & 0.87 & 0.52 \\
10,000 & 0.83 & 0.49 \\
15,000 & 0.81 & 0.48 \\
20,000 & 0.77 & 0.44 \\
25,000 & 0.74 & 0.42 \\
30,000 & 0.73 & 0.40 \\
35,000 & 0.68 & 0.39 \\
40,000 & 0.66 & 0.37 \\
45,000 & 0.61 & 0.36 \\
\hline
\end{tabular}

total walking speed,

$\sum v_{\alpha}=(1.6+1.7+1.7+1.8+2.2+1.1+\cdots)$

$\sum \boldsymbol{v}_{\alpha}=790$

number of agents, $m=500$

average walking speed, $\overline{\boldsymbol{v}}=\frac{\sum \boldsymbol{v}_{\alpha}}{m}$

$\overline{\boldsymbol{v}}=\frac{790}{500}$

$\overline{\boldsymbol{v}}=1.58 \mathrm{~m} / \mathrm{s}$

Since this research is to evaluate the realism of the highdensity crowd simulation, the real walking speed data are necessary for comparison. Thus, this research used the data gathered by [10] which was culled from the Tawaf rituals during Hajj season in 2009. The estimated number of pilgrims during that time approximates 45,000 individuals who journey from all parts of the world to attend.

Figure 22 shows a fundamental diagram, comparing the proposed high-density crowd simulation framework walking speed and the crowd population. The fundamental diagram is one of the methods to validate the realism of the crowd simulation [9, 21, 44, 52]. The fundamental diagram recognized the relationship between pedestrian density and walking speed. The higher the density of the crowd within an area, the slower the average walking speed of the crowd will be. As can be seen in Fig. 22, it shows that the average walking speed of the crowd becomes slower as the population of the crowd become higher. The proposed framework shows a much slower average walking speed because

\section{Fundamental Diagram}

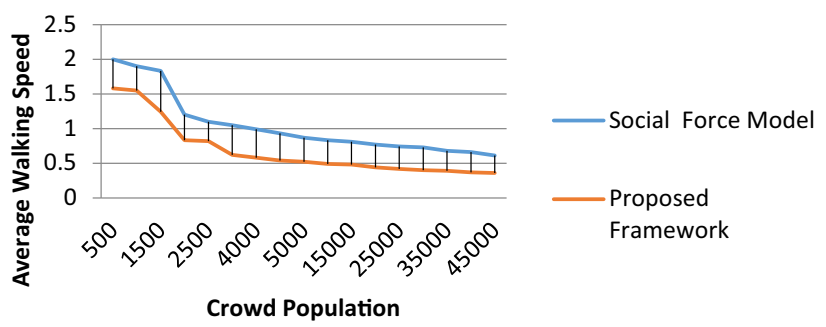

Fig. 22 Comparison between SFM and the proposed method

this framework treats every agent as individuals and causes the crowd flow to become more chaotic and disorganized. As can be seen, the time complexity for the proposed method is the same as the basic SFM, $O\left(n^{2}\right)$; then, there is no concern by increasing the number of agents, as long as there is space between agents to move, the speed will be reduced slightly. In Kaabeh case of study, the limitation depends on the capacity of Tawaf place. Compared to the reality, 45,000 is an appropriate approximation. In general, if there is no limitation for the place, the method behaves as expected. However, if the place is limited, behaviour of the method changes near to the capacity of the place. To clarify this, we have tested the method with some other number of agents close to the capacity of the place and shows that the walking speed reduces to zero when the capacity exceeds.

This simulation deals with the Tawaf ritual as it is considered the densest moving crowd by humans, so far. As the walking speed, intention outlook, gender, and grouping of the agents have been considered, there is no 
difference in other situations with exception of the need for minor adjustment for obstacles. Any changes in terms of the path will not affect the result of the whole method except there are special group movements such as moving in straight lines or creating a specific shape in the group. In these cases, those factors need to be included which currently is out of the scope of this paper.

\section{Conclusion}

In this research, we tried to improve the quality of pedestrian movement behaviour in a high-dense population through microscopic pedestrian approach and crowd control studies. This research has come out with two new concepts to be integrated into the crowd simulation framework. The concept is known as the Zahmah social force model (ZSFM) and the motion stopper model (MSM). These concepts control the behaviour of the pedestrian in a high-density crowd individually but also as a whole. Naturally, these developments lead to two important contributions to pedestrian studies in general: rules following and stopping motion behaviour which are handled by ZSFM and MSM, respectively. Consequently, the combination of both models makes the crowd simulation results more realistic.

ZSFM could produce efficient forces to avoid collision between agents without separating agent whom within a group. In addition, three more characteristics can be realized successfully in this high-density crowd simulation. Those characteristics are intention, walking speed and gender, respectively.

In MSM, there were two types of stopping behaviour being considered: firstly, the sudden stopping which occurs when the pedestrians stop unexpectedly. For example, when one agent walks in front of another agent unexpectedly, it causes the later to stop walking abruptly. Second is the slow stopping behaviour, which happens when the agents slow down before stopping. This scenario mostly happens if an agent follows a group of slow-moving agents. Differences in walking speed amongst the agents will be altered and adapted to the common group's walking speed. The prerequisite to adjust the walking speed entails that the faster agents to slow down and stop for a while before readapting their walking speed.

We discussed how high-density crowd simulation framework works. Then, the framework was evaluated comparing to the real-world scenarios which shows how closely the simulation results to the real-world scenarios in terms of behaviours and walking speed are. This research uses a fundamental diagram to evaluate the realism of the proposed framework. Based on the experimental results, it shows that the proposed framework can simulate the high- density crowd simulation realistically. Hence, it can be concluded that the proposed approach was successful in simulating high-density crowd behaviour such as Tawaf.

\section{Compliance with ethical standards}

Conflict of interest The authors confirm that there is no conflict of interest with this submission and this is an original work.

Open Access This article is licensed under a Creative Commons Attribution 4.0 International License, which permits use, sharing, adaptation, distribution and reproduction in any medium or format, as long as you give appropriate credit to the original author(s) and the source, provide a link to the Creative Commons licence, and indicate if changes were made. The images or other third party material in this article are included in the article's Creative Commons licence, unless indicated otherwise in a credit line to the material. If material is not included in the article's Creative Commons licence and your intended use is not permitted by statutory regulation or exceeds the permitted use, you will need to obtain permission directly from the copyright holder. To view a copy of this licence, visit http://creativecommons. org/licenses/by/4.0/.

\section{Appendix}

List of symbols

\begin{tabular}{ll}
\hline Parameter & Description \\
\hline$\alpha$ & Agent \\
$\overrightarrow{\boldsymbol{p}}_{\alpha}$ & Agent's position \\
$x_{\alpha}$ & $x$-axis agent's position \\
$y_{\alpha}$ & $y$-axis agent's position \\
$\vec{f}_{\text {drive }}$ & Driving force \\
$\vec{f}_{\text {repulsion }}$ & Repulsion force \\
$\vec{f}_{\text {attraction }}$ & Attraction force \\
$f_{\text {social }}$ & Sum of attraction \\
$\overrightarrow{\boldsymbol{d}}_{\alpha}$ & Desired direction of $\alpha$ agent, repulsion and driving \\
$\overrightarrow{\boldsymbol{v}}_{\alpha}$ & Walking velocity for agent $\alpha$ \\
$\boldsymbol{v}_{a}$ & Walking speed for agent $\alpha$ \\
$v_{a}^{d}$ & Deviation of $\boldsymbol{v}_{\alpha}$ \\
$\boldsymbol{d}$ & Distance from the goal \\
$\overrightarrow{\boldsymbol{d}}_{\alpha}$ & Normal distance vector from $\alpha$ to the goal \\
$\overrightarrow{\boldsymbol{v}}_{c}$ & Actual walking velocity \\
$\tau_{a}$ & Relaxation time for agent $\alpha$ \\
$\overrightarrow{\boldsymbol{g}}_{\alpha}$ & Goal position of agent $\alpha$ \\
$r_{\alpha}$ & Radius of interaction for agent $\alpha$ \\
$\overrightarrow{\boldsymbol{n}}_{a \beta}$ & Normalized vector pointing from agent $\alpha$ towards agent \\
$c$ & $\quad$ Scaling parameter of attraction forces between agents \\
$\overrightarrow{\boldsymbol{t}}$ & Epicentre of the circumambulation \\
\hline &
\end{tabular}




\begin{tabular}{ll}
\hline Parameter & Description \\
\hline $\boldsymbol{s}$ & Strength of the repulsion forces \\
$\overline{\boldsymbol{v}}$ & Average walking speed \\
$|v|$ & Magnitude of $v$ \\
\hline
\end{tabular}

\section{References}

1. Abdelghany A, Abdelghany K, Mahmassani H (2016) A hybrid simulation-assignment modeling framework for crowd dynamics in large-scale pedestrian facilities. Transp Res Part A Policy Practice 86:159-176

2. Alqurashi R, Altman T (2019) Hierarchical agent-based modeling for improved traffic routing. Appl Sci 9(20):4376

3. Arab News (2013) Capacity of mataf to reach 105,000 in 2015. Retrieved from Arab news website. http://www.arabnews.com/ news $/ 456434$

4. Bailenson JN, Blascovich J, Beall AC, Loomis JM (2001) Equilibrium theory revisited: mutual gaze and personal space in virtual environments. Presence 10(6):583-598

5. Bian C, Chen D, Wang S (2010) Velocity field based modelling and simulation of crowd in confrontation operations. In: 16th international conference on parallel and distributed systems, pp 646-651

6. Biswal MK (2014) Comparison of pedestrian fundamental diagram: a cultural and gender aspect, MS.c thesis, for the degree of master of technology in transportation engineering, Department of Civil Engineering, National Institute of Technology

7. Boström H, Wensby L (2012) Large-scale agent-based pedestrian and crowd simulation in real-time

8. Chen M, Wang J, Zhi Y, Sun J (2019) Impact of intersecting angles on evacuation efficiency of pedestrian flows in high volume: a case study in metro station. KSCE J Civil Eng 23(5):2324-2332

9. Das JB, Chattaraj U, Nayak S (2016) Studies on pedestrian fundamental diagrams: field observations and controlled experiments. Int J Res Chem Metall Civil Eng 3(1):86-89

10. Dridi MH (2015) List of parameters influencing the pedestrian movement and pedestrian database. Int $\mathbf{J}$ Soc Sci Stud 3(4):94-106

11. Eliyan L, Halabi O, Saleh M (2018) Modeling family behaviour in crowd simulation. In: 2018 international conference on computer and applications (ICCA), pp 66-70. IEEE

12. Erneholm CO (2011) Simulation of the flocking behaviour of birds with the boids algorithm. Royal Institute of Technology

13. Haughton M (2020) Narrativizing Difference in Earlier Bronze Age Society: a comparative analysis of age and gender ideologies in the burials of Ireland and Scotland (Doctoral dissertation, University of Cambridge)

14. Helbing D, Molnár P (1995) Social force model for pedestrian dynamics. Phys Rev E 51(5):5096

15. Ibe N, Masuyama G, Yamashita A, Asama H (2013) Humanlike behaviour model with probabilistic intention. In: ACM SIGGRAPH, Posters, vol 7

16. Johansson A (2004) Pedestrian simulations with the social force model, MS.c thesis. Institute for Transport and Economics, Dresden University of Technology, Germany
17. Jiang Y, Wong SC, Zhang P, Liu R, Duan Y, Choi K (2012) Numerical simulation of a continuum model for bi-directional pedestrian flow. Appl Math Comput 218(10):6135-6143

18. Kang Z, Zhang L, Li K (2019) An improved social force model for pedestrian dynamics in shipwrecks. Appl Math Comput 348:355-362

19. Kapadia M, Singh S, Reinman G, Faloutsos P (2011) A behaviour authoring framework for multi-actor simulations. IEEE Comput Gr Appl 31:45-55. https://doi.org/10.1109/MCG.2011.68

20. Kurdi O, Stannett M, Romano DM (2015) Modeling and simulation of tawaf and sa'yee: a survey of recent work in the field. In: 29th annual European simulation and modelling conference, pp 441-447

21. Lemercier S, Jelic A, Kulpa R, Hua J, Fehrenbach J, Degond P, Appert-Rolland C, Donikian S, Pettré J (2012) Realistic following behaviour $\mathrm{s}$ for crowd simulation. Eurographics 31(2):489-498

22. Lerner A, Chrysanthou Y, Shamir A, Cohen-Or D (2009) Data driven evaluation of crowds. In: Egges A, Geraerts R, Overmars M (eds) MIG, vol 5884, pp 75-83

23. Lim CK, Tan KL, Zaidan AA, Zaidan BB (2020) A proposed methodology of bringing past life in digital cultural heritage through crowd simulation: a case study in George Town, Malaysia. Multimed Tools Appl 79(5):3387-3423

24. Liu J, Chen X (2019) Simulation of passenger motion in metro stations during rush hours based on video analysis. Autom Constr 107:102938

25. Li Q, Liu Y, Kang Z, Li K, Chen L (2020) Improved social force model considering conflict avoidance. Chaos Interdiscip J Nonlinear Sci 30(1):013129

26. Loscos C, Marchal D, Meyer A (2003) Intuitive crowd behaviour in dense urban environments using local laws

27. Mao Y, Yang S, Li Z, Li Y (2018) Personality trait and group emotion contagion based crowd simulation for emergency evacuation. Multimed Tools Appl 5:1-28

28. Moussaïd M, Nelson JD (2014) Simple heuristics and the modelling of crowd behaviours. In: Pedestrian and evacuation dynamics, pp 75-90. Springer, Cham

29. Noh B, Youm C, Lee M, Park H (2020) Age-specific differences in gait domains and global cognitive function in older women: gait characteristics based on gait speed modification. PeerJ 8:e8820

30. Pelechano GN (2006) Modeling realistic high density autonomous agent crowd movement: social forces, communication, roles and psychological influences

31. Peng Y, Li SW, Hu ZZ (2019) A self-learning dynamic path planning method for evacuation in large public buildings based on neural networks. Neurocomputing 5:98

32. Pinna F, Murrau R (2018) Age factor and pedestrian speed on sidewalks. Sustainability 10(11):4084

33. Ren Z, Charalambous P, Bruneau J, Peng Q, Pettré J (2016) Group modeling: a unified velocity-based approach. Comput $\mathrm{Gr}$ Forum 2:926

34. Rincon JA, Costa A, Villarrubia G, Julian V, Carrascosa C (2018) Introducing dynamism in emotional agent societies. Neurocomputing 272:27-39

35. Salehi N, Sung M (2020) Realistic multi-agent formation using discretionary group behaviour (DGB). Appl Sci 10(10):3518

36. Sarmady S, Haron F, Talib AZ (2011) A cellular automata model for circular movements of pedestrians during Tawaf. Simul Model Pract Theory 19(3):969-985

37. Sticco IM, Frank GA, Dorso CO (2020) Social force model parameter testing and optimization using a high stress real-life situation. arXiv preprint arXiv:2007.06651 
38. Strnad D, Nerat A, Kohek Š (2017) Neural network models for group behaviour prediction: a case of soccer match attendance. Neural Comput Appl 28(2):287-300

39. Strickland BB (2001) Gale encyclopedia of psychology, 2nd edn. Gale Group, Detroit, MI

40. Trautman P, Patel K (2020) Real time crowd navigation from first principles of probability theory. In: Proceedings of the international conference on automated planning and scheduling, Vol 30, pp 459-467

41. Ullah H, Ullah M, Uzair M (2018) A hybrid social influence model for pedestrian motion segmentation. Neural Comput Appl 96:1-17

42. Wang J, Chen M, Jin B, Li J, Wang Z (2019) Propagation characteristics of the pedestrian shockwave in dense crowd: experiment and simulation. Int J Disaster Risk Reduct 40:101287

43. Wang J, Jin B, Li J, Chen F, Wang Z, Sun J (2019) Method for guiding crowd evacuation at exit: the buffer zone. Saf Sci 118:88-95

44. Wolinski D, Guy SJ, Olivier A-H, Lin M, Manocha D, Pettre J (2014) Parameter estimation and comparative evaluation of crowd simulations. Comput Gr Forum 33:303-312

45. Xu M, Wu Y, Lv P, Jiang H, Luo M, Ye Y (2015) miSFM: on combination of mutual information and social force model towards simulating crowd evacuation. Neurocomputing 168:529-537
46. Yao Z, Zhang G, Lu D, Liu H (2020) Learning crowd behaviour from real data: a residual network method for crowd simulation. Neurocomputing 2:1069

47. YouTube (2019) Hajj 2012 Tawaf of kaaba. Retrieved from YouTube website. https://www.youtube.com/watch?v= 2SOWVV5Ez3k

48. Zhang J, Liu H, Li Y, Qin X, Wang S (2018) Video-driven group behaviour simulation based on social comparison theory. Phys A Stat Mech Appl 512:620-634

49. Zhao M, Zhong J, Cai W (2018) A role-dependent data-driven approach for high-density crowd behaviour modeling. ACM Trans Model Comput Simul 28(4):1-25

50. Zainuddin Z, Thinakaran K, Shuaib M (2010) Simulation of the pedestrian flow in the Tawaf Area using the social force model. World Acad Sci Eng Technol 1(50):910

51. Zhou S, Chen D, Cai W, Luo L, Low MYH, Tian F, Tay VS-H, Ong DWS, Hamilton BD (2010) Crowd modeling and simulation technologies. ACM Trans Model Comput Simul 20:4

52. Zwan M (2016) The impact of density measurement on the fundamental diagram. MS.c thesis, Technical Artificial Intelligence. University of Utrecht, the Netherlands

Publisher's Note Springer Nature remains neutral with regard to jurisdictional claims in published maps and institutional affiliations. 